Research Article

\title{
BLSTM-Based Adaptive Finite-Time Output-Constrained Control for a Class of AUSs with Dynamic Disturbances and Actuator Faults
}

\author{
Shiyi Huang $\mathbb{D}^{1}{ }^{1}$ Lulu Rong, ${ }^{2}$ Xiaofei Chang, ${ }^{3}$ Zheng Wang $\mathbb{D}^{3,4,5}$ Zhaohui Yuan, ${ }^{1}$ \\ and Caisheng Wei $\mathbb{1}^{6}$ \\ ${ }^{1}$ Software Institute, East China Jiaotong University, Nanchang, China \\ ${ }^{2}$ School of Astronautics, Northwestern Polytechnical University, Xi'an 710072, China \\ ${ }^{3}$ National Key Laboratory of Aerospace Flight Dynamics, Northwestern Polytechnical University, Xi'an, China \\ ${ }^{4}$ Research Center for Unmanned System Strategy Development, Northwestern Polytechnical University, Xi'an, China \\ ${ }^{5}$ Unmanned System Research Institute, Northwestern Polytechnical University, Xi'an, China \\ ${ }^{6}$ School of Aeronautics and Astronautics, Central South University, Changsha 410083, China \\ Correspondence should be addressed to Zheng Wang; wz_nwpu@126.com and Caisheng Wei; caisheng_wei@csu.edu.cn
}

Received 11 May 2021; Revised 9 September 2021; Accepted 15 September 2021; Published 23 September 2021

Academic Editor: Omar-Jacobo Santos

Copyright (c) 2021 Shiyi Huang et al. This is an open access article distributed under the Creative Commons Attribution License, which permits unrestricted use, distribution, and reproduction in any medium, provided the original work is properly cited.

\begin{abstract}
In this paper, a BLSTM-based adaptive finite-time control structure has been constructed for a class of aerospace unmanned systems (AUSs). Firstly, a novel neural network structure possessing both the time memory characteristics and high learning speed, broad long short-term memory (BLSTM) network, has been constructed. Secondly, several nonlinear functions are utilized to transform the tracking errors into a novel state vector to guarantee the output constraints of the AUSs. Thirdly, the fractionalorder control law and the corresponding adaptive laws are designed, and as a result, the adaptive finite-time control scheme has been formed. Moreover, to handle the uncertainties and the faulty elevator outputs, an inequality of the multivariable systems is utilized. Consequently, by fusing the output of the BLSTM, the transformation of the tracking errors, and the adaptive finite-time control law, a novel BLSTM-based intelligent adaptive finite-time control structure has been established for the AUSs under output constraints. The simulation results show that the proposed BLSTM-based adaptive control algorithm can achieve favorable control results for the AUSs with multiple uncertainties.
\end{abstract}

\section{Introduction}

As is well known, the adaptive control methodology can adjust control parameters automatically according to the variation of the environment and the system uncertainties and can improve the adaptability with respect to the uncertain factors mentioned above [1-5]. In recent years, a lot of research has been done on adaptive control in China. In [6], a novel adaptive switching dynamic surface control (DSC) strategy is first presented for fractional-order nonlinear systems in the nonstrict feedback form with unknown dead zones and arbitrary switching. In [6], a novel adaptive prescribed performance tracking control scheme has been proposed for stochastic nonlinear systems with unmodeled dynamics in finite-time prescribed performance. In [7], a novel event-triggered-based fuzzy adaptive finite-time control method has been proposed for stochastic nonlinear nonstrict feedback systems with unmodeled dynamics. In [8], a novel fault-tolerant IGC structure has been constructed for a class of skid-to-turn (STT) missiles subjected to rapidly changing actuator failures and coupled multisource uncertainties. In [9], a novel adaptive fault-tolerant attitude control approach has been designed based on the long short-term memory (LSTM) network for the fixedwing UAV subject to the high dynamic disturbances and actuator faults. In [10], a novel evasion-faced fast adaptive 
feed-forward control structure has been established for the hypersonic vehicles. Adaptive robust control is also a typical representative of adaptive control methodology, which can effectively realize the stable control of the system. In [11], a vehicle mass estimation algorithm based on the least squares method and sliding mode observer is proposed for variable mass unmanned aircraft systems. In [12], the fuzzy neural network (FNN) control method using impedance learning has been proposed for coordinated multiple constrained robots carrying a common object. In [13], the adaptive control-based design strategies have been proposed for a pressurized water (PWR) nuclear power plant (NPP) system. In [14], a reinforcement learning (RL) structure control strategy based on actor-critic has been developed for a flexible two-link manipulator (FTLM) system. In [15], robust fault-tolerant control for a class of second-order nonlinear systems using an adaptive third-order sliding mode control was proposed. In [16], an adaptive control scheme under the hierarchical framework has been proposed for the trajectory tracking problem of flapping-wing microaerial vehicles (FWMAVs). In [17], the adaptive robust tracking control scheme based on backstepping technique has been proposed for a class of multi-input and multioutput (MIMO) nonaffine systems with uncertain structure and parameters, unknown control direction, and unknown external disturbance. Furthermore, the adaptive control is usually used together with some other control laws, inheriting the original advantages of these control laws, as well as taking advantages of the automatic regulation of the adaptive control [18-20]. In practice, since the attitude control performance of the aerospace unmanned systems is always affected by aerodynamic uncertainties and external disturbances, the adaptive control method has been introduced into the control design process of AUS. For instance, the adaptive sliding mode control $[21,22]$, adaptive fuzzy control [23-25], model reference adaptive control (MRAC) [26], adaptive dynamic inversion control [27], adaptive backstepping control [28-30], adaptive super-twisting approach [31], and adaptive anti-disturbance control [32, 33] have all been applied to the attitude control design of the AUSs. Nevertheless, it is difficult to utilize the aforementioned control technique to guarantee rapidity of the closed-loop control system, especially when the external circumstance is fickle.

In order to tackle this problem, the concept of finite-time control (FTC) was put forward in the middle of the last century [34-36]. Given a limit condition of the system initial value, if the system states never exceed a certain threshold within a finite-time interval, the system is described as finitetime stable. In the past decade, many studies have been built on infinite time asymptotic convergence and can be utilized to guarantee the system convergence under the fickle external circumstance [37-40]. Due to the outstanding advantages under time-constraint situations, a series of papers have explored the potential of finite-time control laws. In [37], the finite-time Lyapunov stability theorem has been established. In [38], through output feedback based on a finite-time separation principle, the global finite-time stability has been realized for the double integrator system. In [39], a finite-time adaptive controller has been designed for a class of strict feedback nonlinear systems. Moreover, by the combination of a robust controller [40] and an adaptive controller [41], the finite-time tracking task was completed and the difficulties caused by state constraints and dead zone were conquered. In addition, FTC has also been employed in space missions. In [42], the finite-time stability of spacecraft large-angle maneuver under disturbances was achieved, avoiding the chattering phenomenon by virtue of a robust adaptive controller. In [43], a finite-time control strategy based on a finite-time auxiliary system has been designed for a 12-rotor UAV. Compared with the finite-time control law, the fixed-time control law has the outstanding advantage in the specific situation, and it can effectively realize the stable control at a given time. In [44], the event-triggered fixedtime adaptive fuzzy control method has been proposed for state-constrained stochastic nonlinear systems. In [45], a novel adaptive fixed-time control strategy has been proposed for a class of nonlinear bilateral teleoperation systems. In [46], a light fixed-time controller has been designed for the issues of chattering effects and multiple parameters.

Moreover, intelligent methods, algorithms, and theories have achieved remarkable progress recently, providing a useful way to improve the response speed and the tracking performance of the control systems [47-50]. The broad learning system (BLS), which utilizes the self-adjusting mechanism of node number to achieve better learning performance, has been firstly proposed in [51]. In [52], based on the broad learning method, a framework that can learn and fuse two modal characteristics has been constructed, possessing better stability and rapidity. Combining the Takagi-Sugeno (TS) fuzzy modeling method with the BLS, the fuzzy broad learning system (BLS) has been established in [53], which shows advantages over neuro-fuzzy models. By combining a convolutional neural network with broad learning system, a framework for license plate recognition has been reported in [54]. In [55], the least p-norm-based BLS (LP-BLS) using the fixed-point iteration strategy has been proposed. Furthermore, BLS has also been used to solve practical engineering problems [56-58]. In [56], a novel quaternion broad learning system has been proposed. In [57], the BLS has been introduced into hyper-spectral image analysis area, providing new ideas and technical reserves for a variety of hyper-spectral image analysis problems. Based on BLS, Zhongdong et al. [58] proposed a method of landscape capacity allocation and obtained a capacity allocation result that met the total investment cost and minimized network active power loss. Moreover, in [59], the long short-term memory (LSTM) network, which possesses the storage functions and can effectively approximate the time accumulative nonlinearities in the control systems, has been established. In $[60,61]$, the effectiveness and robustness of the LSTM have been demonstrated. In [62], a LSTM-based intelligent control structure is constructed, showing the better approximating accuracy of the LSTM compared to the common neural networks.

However, up to know, the broad learning methods have rarely been introduced into the control design of the AUSs. According to $[51,52]$, the broad learning methods could be made use of to enhance the control performance and reduce the control complexity. Therefore, it is of remarkable importance to 
develop the BLS-based adaptive control methods for the AUSs. Furthermore, in the actual flight process, the nonlinearities and uncertainties of the AUSs usually possess time-varying features and accumulative effect, and as a result, the LSTM should be introduced together with the BLS simultaneously.

Therefore, in this work, we propose a BLSTM-based adaptive finite-time control structure for the AUSs under output constraints. The broad long short-term memory network, which possesses both the time memory characteristics and high learning speed, has been constructed. The fractional-order control law and the tracking error transformation are utilized to guarantee the finite-time convergence and output constraints. Compared with the existing results, this work possesses the following features:

(i) It is the first BLSTM-based adaptive finite-time control structure for the AUSs.

(ii) The proposed broad long short-term memory network can be applied to a plenty of practical engineering systems to improve the approximation, classification, or recognition performance.

(iii) By introducing the BLSTM into the adaptive finitetime control law, the convergence time can be ensured and the control accuracy can be improved.

\section{Problem Formulation and Preliminaries}

2.1. The System Model of Aerospace Unmanned System. According to reference [63], the kinematic and dynamic equations of the aerospace unmanned system can be expressed by

$$
\begin{aligned}
& \dot{\vartheta}=\omega_{y} \sin \gamma+\omega_{z} \cos \gamma . \\
& \dot{\psi}=\frac{\left(\omega_{y} \cos \gamma-\omega_{z} \sin \gamma\right)}{\cos \vartheta}, \\
& \dot{\gamma}=\omega_{x}-\tan \vartheta\left(\omega_{y} \cos \gamma-\omega_{z} \sin \gamma\right), \\
& \dot{\omega}=J^{-1}[M-\omega \times(J \cdot \omega)],
\end{aligned}
$$

where $\vartheta, \psi$, and $\gamma$ denote the pitch angle, yaw angle, and roll angle, respectively; $\omega$ is the attitude angular velocity; $I$ is the inertia matrix of the AUS; and $M$ is the total moment of force.

$$
\begin{aligned}
I & =\left[\begin{array}{ccc}
I_{x x} & 0 & I_{x z} \\
0 & I_{y y} & 0 \\
I_{z x} & 0 & I_{z z}
\end{array}\right], \\
M & =q S L\left[\begin{array}{c}
C_{z} \\
C_{y} \\
C_{x}
\end{array}\right], \\
C_{z} & =C_{z 0}+C_{z}^{\delta_{a 1}} \delta_{a 1}+C_{x}^{\delta_{a 2}} \delta_{a 2}+C_{z}^{\delta_{b 1}} \delta_{b 1}+C_{z}^{\delta_{b 2}} \delta_{b 2}+C_{z}^{\alpha} \alpha, \\
C_{y} & =C_{y}^{\delta_{y}} \delta_{y}+C_{y}^{\beta} \beta, \\
C_{x} & =C_{x}^{\delta_{a 1}} \delta_{a 1}+C_{x}^{\delta_{a 2}} \delta_{a 2}+C_{x}^{\delta_{b 1}} \delta_{b 1}+C_{x}^{\delta_{b 2}} \delta_{b 2}+C_{x}^{\beta} \beta .
\end{aligned}
$$

The definitions of $q, \rho, V, S, L, C_{z}, C_{y}, C_{x}, C_{z 0}, \delta_{a 1}, \delta_{a 2}$, $\delta_{b 1}, \delta_{b 2}$, and $\delta_{y}$ can be found in [64]. $\alpha, \beta$ are the angle of attack and sideslip angle, respectively, with the following dynamic equations:

$$
\begin{aligned}
& \dot{\alpha}=\omega_{z}+\frac{\rho V S C_{z \alpha}}{2 m} \alpha, \\
& \dot{\beta}=-\omega_{y}+\frac{\rho V S C_{y \beta}}{2 m} \beta .
\end{aligned}
$$

With the external disturbances, the attitude dynamics of the AUS system can be modeled as

$$
\begin{aligned}
& \dot{\theta}(t)=A_{\theta} \omega(t)+d_{\theta}(t), \\
& \dot{\omega}(t)=B_{\omega} \delta(t)+C(\omega)+d_{\omega}(t),
\end{aligned}
$$

where $\theta(t)=\vartheta, \psi, \gamma^{T}$ and $\omega(t)=\left[\omega_{z}, \omega_{y}, \omega_{x}\right]^{T}$; $\delta(t) e=\left[\delta_{b 1}, \delta_{b 2}, \delta_{y}, \delta_{a 1}, \delta_{a 2}\right]^{T} ; y_{\text {out }}$ is the output of the AUS system; and $d_{\theta}(t)$ and $d_{\omega}(t) \in \mathbb{R}^{3 \times 1}$ are the matched and mismatched disturbances.

$$
\begin{aligned}
A_{\theta} & =\left[\begin{array}{ccc}
\cos \gamma & \sin \gamma & 0 \\
-\frac{\sin \gamma}{\cos \vartheta} & \frac{\cos \gamma}{\cos \vartheta} & 0 \\
\tan \vartheta \sin \gamma & -\tan \vartheta \cos \gamma & 1
\end{array}\right], \\
B_{\omega} & =q S L I^{-1} C_{M}, \\
C(\omega) & =I^{-1}\left\{\begin{array}{c}
C_{z 0}+C_{z}^{\alpha} \alpha \\
C_{y}^{\beta} \beta \\
C_{x}^{\beta} \beta
\end{array}\right]+\left[\begin{array}{c}
\omega_{z} \\
\omega_{y} \\
\omega_{x}
\end{array}\right] \times\left(I\left[\begin{array}{c}
\omega_{z} \\
\omega_{y} \\
\omega_{x}
\end{array}\right]\right),
\end{aligned}
$$

where

$$
C_{M}=\left[\begin{array}{ccccc}
C_{z}^{\delta_{b 1}} & C_{z}^{\delta_{b 2}} & 0 & C_{z}^{\delta_{a 1}} & C_{z}^{\delta_{a 2}} \\
0 & 0 & C_{y}^{\delta_{y}} & 0 & 0 \\
C_{x}^{\delta_{b 1}} & C_{x}^{\delta_{b 2}} & 0 & C_{x}^{\delta_{a 1}} & C_{x}^{\delta_{a 2}}
\end{array}\right] .
$$

Considering these factors, there often exist structural uncertainties and modeling errors, which can be rewritten as

$$
\begin{aligned}
\dot{\theta}(t) & =\left[A_{\theta}+\Delta A_{\theta}\right] \omega(t)+f(\theta)+d_{\theta}(t), \\
\dot{\omega}(t) & =\left[B_{\omega}+\Delta B_{\omega}\right] \delta(t)+C(\omega)+f(\theta, \omega)+d_{\omega}(t),
\end{aligned}
$$

where $f(\theta), f(\theta, \omega)$ represent the unknown lumped aerodynamic uncertainties. Let $\Delta A_{\theta}$ represent the structural uncertainty of $A_{\theta}$ and let $\Delta B_{\omega}$ represent the composite uncertainties.

$$
\Delta B_{\omega}=\zeta \cdot \Delta C_{M}+\Delta \zeta \cdot C_{M},
$$

where $\Delta \zeta$ is the uncertainty of $\zeta$.

Therefore, the control objective of this paper is to force the attitude angle $\theta$ to follow the desired signals in spite of 
the structural uncertainties and the aerodynamic uncertainties.

\subsection{Preliminaries}

Assumption 1. It is supposed that $A_{\theta}$ and $B_{\omega}$ are invertible or generalized invertible.

Assumption 2. It is supposed that $d_{\theta}(t), d_{\omega}(t)$ are both bounded, e.g., there exist unknown positive constants $\bar{D}_{\theta}$ and $\bar{D}_{\omega}$ such that $\left\|d_{\theta}(t)\right\| \leq \bar{D}_{\theta},\left\|d_{\omega}(t)\right\| \leq \bar{D}_{\omega}$.

The following lemmas are required.

Lemma 1 (see [65]). Given any $a_{1}, a_{2}$ and positive constants $z_{1}, z_{2}, z_{3}$, the following inequality can be satisfied:

$$
\left|a_{1}\right|^{z_{1}}\left|a_{2}\right|^{z_{2}} \leq \frac{z_{1}}{z_{1}+z_{2}} z_{3}\left|a_{1}\right|^{z_{1}+z_{2}}+\frac{z_{2}}{z_{1}+z_{2}} z_{3}^{-z_{1} / z_{2}}\left|a_{2}\right|^{z_{1}+z_{2}} .
$$

Lemma 2 (see [66]). Given any constant $\varepsilon>0$ and any variable $x \in \mathbb{R}$, the following inequality can be satisfied:

$$
0<\|x\|-\frac{x^{T} x}{\sqrt{x^{T} x+\varepsilon}}<\varepsilon .
$$

Lemma 3 (see [42]). Consider system $\dot{X}=f(X, U)$. Given any two differential positive definite functions $V(X)$ and $c>0,0<l<1, \varepsilon>0$ such that

$$
\dot{V}(X) \leq-c V^{l}(X)+\varepsilon, \quad t \geq 0,
$$

the nonlinear system $\dot{X}=f(X, U)$ is semi-globally practical finite-time stable (SGPFS).

\subsection{Broad Long Short-Term Memory (BLSTM) Network.} The structure of BLSTM network is presented in Figure 1.

Note that the broad learning thought has been introduced to build the structure of BLSTM, and the concept of "gate" is inherited. In BLSTM, the input data are $\left[h_{t-1}, x_{t}\right]$, and $\sigma\left(W_{1 f i}\left[h_{t-1}+x_{t}\right]+b_{1 f i}\right), i=1,2, \ldots, n$, is utilized to generate the $i$ th mapped features, $Z_{t i}$, where $W_{f i}$ is the random weights with the proper dimensions. Denote $Z^{i} \equiv\left[Z_{t 1}, \ldots, Z_{t i}\right]$, which is the concatenation of all the first $i$ groups of mapping features. Similarly, the $j$ th group of enhancement nodes, $\sigma\left(\left[Z_{t 1} Z_{t 2}, \ldots, Z_{t n}\right] W_{2 f j}+b_{2 f j}\right)$, $j=1,2, \ldots, N$, is denoted as $H_{t j}$, and the concatenation of all the first $j$ groups of enhancement nodes is denoted as $H^{j}=\left[H_{t 1}, \ldots, H_{t j}\right]$. Hence, the broad model of forget gate $f_{t}$ can be represented as the equation of the form

$$
\begin{aligned}
f_{t} & =\left[Z_{t 1}, \ldots, Z_{t n} \mid \sigma\left(Z_{t}^{1} W_{2 f 1}+b_{2 f 1}\right), \ldots, \sigma\left(Z_{t}^{n} W_{2 f n}+b_{2 n}\right)\right] W^{1} \\
& =\left[Z_{t 1}, \ldots, Z_{t n} \mid H_{t 1}, \ldots, H_{t m}\right] W^{1} \\
& =\left[Z_{t n} \mid H_{t m}\right] W^{1},
\end{aligned}
$$

where $W^{1}=\left[Z_{t n} \mid H_{t m}\right]^{+} f_{t}$ represents the connecting weights for the broad structure. Similarly, we can get the broad model of memory state $\widetilde{c}_{t}$ as $\widetilde{c}_{t}=\left[Q_{t n} \mid R_{t m}\right] W^{2}$, where $W^{1}=\left[Q_{t n} \mid R_{t m}\right]^{+} \widetilde{c}_{t}$. The mathematical expressions of input gate, update gate, and output gate are

$$
\begin{aligned}
i_{t} & =\sigma\left(W_{i}\left[h_{t-1}, x_{t}\right]+b_{i}\right), \\
c_{t} & =f_{t} \circ c_{t-1}+i_{t} \circ \widetilde{c}_{t}, \\
o_{t} & =\sigma\left(W_{o}\left[h_{t-1}, x_{t}\right]+b_{o}\right), \\
h_{t} & =o_{t} \circ \tanh \left(c_{t}\right),
\end{aligned}
$$

where $W_{f}, W_{i}, W_{c}, W_{o}, b_{f}, b_{i}, b_{c}$, and $b_{o}$ are the weight matrices and $\circ$ is the Hadamard product.

\section{Main Results}

In this section, the controller design will be divided into inner loop and outer loop of AUS subject to finite-time convergence, respectively. In the inner loop, several nonlinear functions are used to constrain the state error to predefined boundness. In view of the external disturbances, the BLSTM network is introduced to approximate the unknown nonlinear functions. The adaptive laws are designed to compensate the structural uncertainties. Meanwhile, in the outer loop, an innovative adaptive finite-time control law is designed to deal with the input uncertainties. The structure of the proposed BLSTM-based intelligent adaptive control method is depicted in Figure 2.

3.1. BLSTM-Based Inner Loop Finite Control Law. Define $e_{1, \vartheta}=\vartheta-\vartheta_{d}, e_{1, \psi}=\psi-\psi_{d}, e_{1, \gamma}=\gamma-\gamma_{d}$. Therefore, the inner loop error dynamic equation can be given by

$$
\dot{e}_{1}=A_{\theta} \Omega_{\theta} \omega+f(\theta)+d_{\theta}(t)-\dot{\theta}_{d},
$$

where $\Omega_{\theta}=\left(A_{\theta}+\Delta A_{\theta}\right) / A_{\theta}$.

The following nonlinear functions are utilized to guarantee the output constraints:

$$
\begin{aligned}
& s_{\vartheta}=\log \frac{\ell_{a, 1}+e_{1,9}}{\ell_{a, 2}+e_{1, \vartheta}} \\
& s_{\psi}=\log \frac{\ell_{b, 1}+e_{1, \psi}}{\ell_{b, 2}+e_{1, \psi}}, \\
& s_{\gamma}=\log \frac{\ell_{c, 1}+e_{1, \gamma}}{\ell_{c, 2}+e_{1, \gamma}} .
\end{aligned}
$$

Define $s_{\theta}=\left[s_{\vartheta}, s_{\psi}, s_{\gamma}\right]^{T}$. Accordingly, we can get that

$$
\dot{s}_{\theta}=T(\theta)\left(A_{\theta} \Omega_{\theta} \omega+f(\theta)+d_{\theta}(t)-\dot{\theta}_{d}\right)
$$

where

$$
T(\theta)=\operatorname{Diag}\left[\frac{e^{s_{\vartheta}}+e^{-s_{\vartheta}}+2}{\ell_{a, 1}+\ell_{a, 2}} \frac{e^{s_{\psi}}+e^{-s_{\psi}}+2}{\ell_{b, 1}+\ell_{b, 2}} \frac{e^{s_{\gamma}}+e^{-s_{\gamma}}+2}{\ell_{c, 1}+\ell_{c, 2}}\right] .
$$




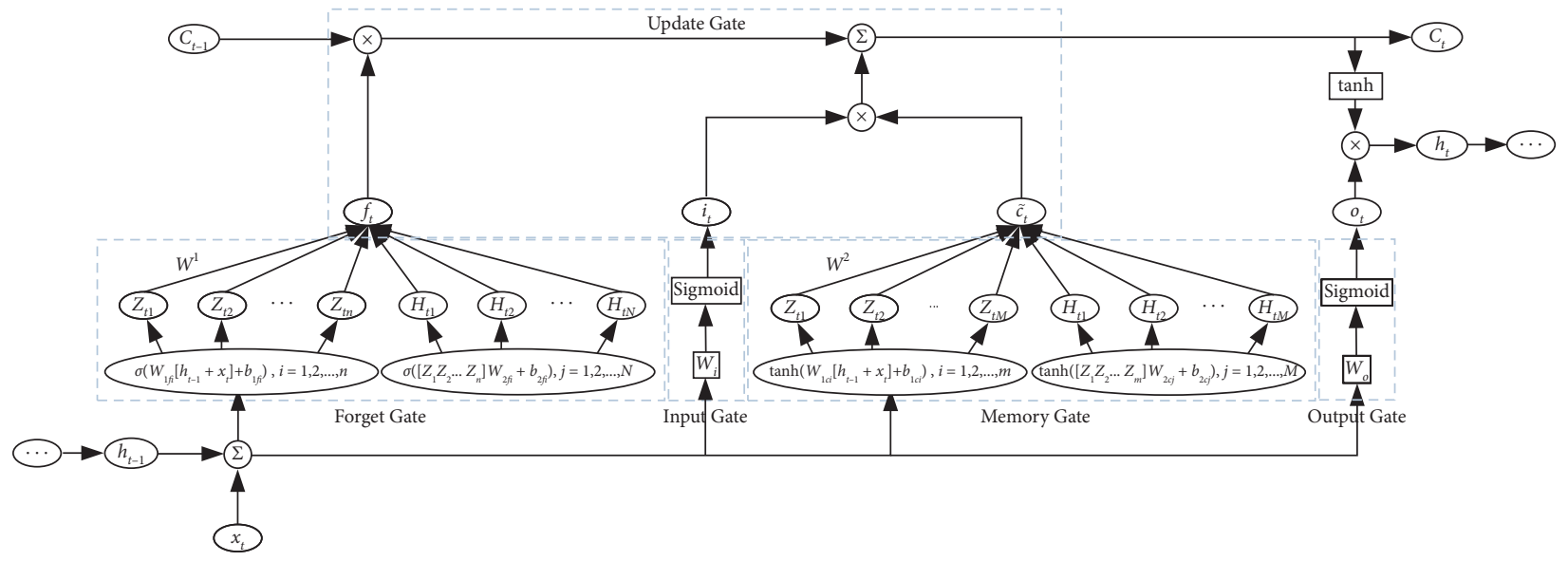

FIgURE 1: The structure of BLSTM.

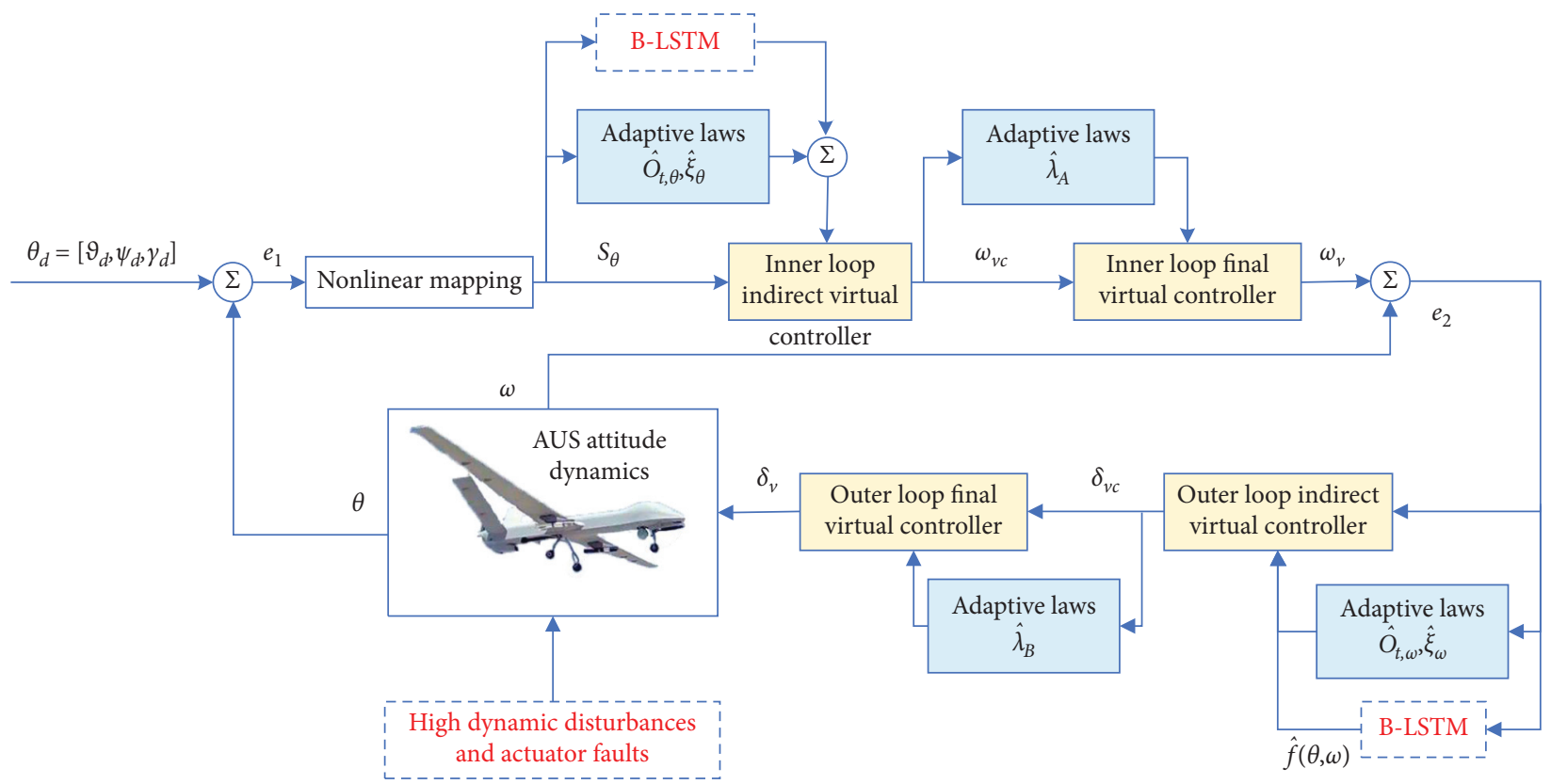

FIgURE 2: The structure of the proposed control scheme.

Considering the uncertainties $f(\theta)$, a BLSTM network $O_{t, \theta} \tanh \left(C_{t, \theta}\right)$ is applied to the inner controller. Clearly, $f(\theta)=O_{t, \theta} \circ \tanh \left(C_{t, \theta}\right)+\varepsilon_{\theta}$, where $\varepsilon_{\theta}$ is the bounded approximation error. Define the BLSTM node number in the inner loop as $N_{1}$. We can design the self-adjust strategy for the nodes number as

$$
\begin{cases}N_{1}=N_{1}+n_{1 \text { ad }}, & \int_{t_{1}-1}^{t_{1}}\left\|s_{\theta}(\tau)\right\| \mathrm{d} \tau>\chi_{1 \text { up }}, \\ N_{1}=N_{1}, & \chi_{1 \text { low }} \leq \int_{t_{1}-1}^{t_{1}}\left\|s_{\theta}(\tau)\right\| \mathrm{d} \tau \leq \chi_{1 \text { up }}, \\ N_{1}=N_{1}-n_{1 \text { ad }}, & \int_{t_{1}-1}^{t_{1}}\left\|s_{\theta}(\tau)\right\| \mathrm{d} \tau<\chi_{\text {llow }},\end{cases}
$$

where $n_{1 \text { ad }}$ represents the adjustment and it is a designed positive integer; $\chi_{1 \text { low }}, \chi_{1 \text { up }}$ are designed positive constants, denoting the adjustment threshold; $t_{1}$ represents the time series to complete the judgment and adjustment; and $\widehat{O}_{t, \theta}$ is the estimated value of $O_{t, \theta}$.

Define $\xi_{\theta}=\sup _{t \geq 0}\left\|\varepsilon_{\theta}+d_{\theta}(t)\right\|$. Considering (16), the indirect virtual control signal is designed as

$$
\begin{aligned}
& \omega_{v c}=A_{\theta}^{-1}\left(-\frac{k_{1} s_{\theta}{ }^{2 l-1}}{T(\theta)}-\widehat{O}_{t, \theta} \tanh \left(C_{t, \theta}\right)-\widehat{\xi}_{\theta} \varphi(\theta)+\dot{\theta}_{d}\right), \\
& \varphi(\theta)=\frac{\Lambda^{T}(\theta)}{\sqrt{\Lambda(\theta) \Lambda^{T}(\theta)+\varepsilon_{\xi, \Lambda}^{2}}}, \quad \forall \varepsilon_{\xi, \Lambda}>0,
\end{aligned}
$$

where $\omega_{v c}$ is the virtual control signal of $\omega, k_{1} \in \mathbb{R}^{3 \times 3}$ is the control gain, $\widehat{\xi}_{\theta}$ is the estimated value of $\xi_{\theta}$, and $\Lambda(\theta)=\left(s_{\theta}^{T} s_{\theta}\right) s_{\theta}^{T} T(\theta)$.

Define $\hat{\lambda}_{A}$ as the estimated value of $\lambda_{A}$. Therefore, we design the inner control law as 


$$
\omega_{v}=-\frac{\hat{\lambda}_{A} \omega_{v c}\left(\Upsilon(\theta) \hat{\lambda}_{A} \omega_{v c}\right)}{\sqrt{\left(\Upsilon(\theta) \hat{\lambda}_{A} \omega_{v c}\right)^{2}+\varepsilon_{A}^{2}}}, \quad \forall \varepsilon_{A}>0,
$$

where $\Upsilon(\theta)=\Lambda(\theta), A_{\theta} \in \mathbb{R}^{1 \times 3}$. Substituting (19) into (16) generates

$$
\begin{aligned}
\dot{s}_{\theta}= & -k_{1} s_{\theta}^{2 l-1}+T(\theta)\left(A_{\theta} \Omega_{\theta} e_{2}+A_{\theta} \Omega_{\theta} \omega_{v}-A_{\theta} \omega_{v c}\right. \\
& \left.-\widetilde{O}_{t, \theta} \tanh \left(C_{t, \theta}\right)-\widehat{\xi}_{\theta} \varphi(\theta)+\varepsilon_{\theta}+d_{\theta}(t)\right),
\end{aligned}
$$

where $e_{2}=\omega-\omega_{v}$.

The following Lyapunov function is selected:

$$
V_{1}=\frac{1}{2} s_{\theta}^{T} s_{\theta}+\frac{1}{2 \Gamma_{O_{t, \theta}}} \widetilde{O}_{t, \theta}^{T} \widetilde{O}_{t, \theta}+\frac{1}{2 \Gamma_{\xi_{\theta}}} \widetilde{\xi}_{\theta}^{2}+\frac{1}{2 \Gamma_{\lambda_{A}} \lambda_{A}} \widetilde{\lambda}_{A}^{2},
$$

where $\widetilde{O}_{t, \theta}=\widehat{O}_{t, \theta}-O_{t, \theta}, \widetilde{\xi}_{\theta}=\widehat{\xi}_{\theta}-\xi_{\theta}, \widetilde{\lambda}_{A}=\widehat{\lambda}_{A}-\lambda_{A}$, and $\Gamma_{O_{t \theta}}$, $\Gamma_{\xi_{\theta}}, \Gamma_{\lambda_{A}}>0$ is the adaptive gain. Take the differential of $V_{1}$ as follows:

$$
\dot{V}_{1}=s_{\theta}^{T} \dot{s}_{\theta}+\frac{1}{\Gamma_{O_{t, \theta}}} \widetilde{O}_{t, \theta}^{T} \dot{\hat{O}}_{t, \theta}+\frac{1}{\Gamma_{\xi_{\theta}}} \tilde{\xi}_{\theta} \dot{\hat{\xi}}_{\theta}+\frac{1}{\Gamma_{\lambda_{A}} \lambda_{A}} \widetilde{\lambda}_{A} \dot{\hat{\lambda}}_{A} .
$$

Substituting (21) into (23) yields

$$
\begin{aligned}
\dot{V}_{1}= & -k_{1} s_{\theta}^{T} s_{\theta}^{2 l-1}+\Lambda(\theta)\left(\begin{array}{c}
A_{\theta} \Omega_{\theta} e_{2}+A_{\theta} \Omega_{\theta} \omega_{v}-A_{\theta} \omega_{v c} \\
-\widetilde{O}_{t, \theta} \tanh \left(C_{t, \theta}\right)-\widehat{\xi}_{\theta} \varphi(\theta)+\varepsilon_{\theta}+d_{\theta}(t)
\end{array}\right) \\
& +\frac{1}{\Gamma_{O_{t, \theta}}} \widetilde{O}_{t, \theta}^{T} \dot{\hat{O}}_{t, \theta}+\frac{1}{\Gamma_{\xi_{\theta}}} \widetilde{\xi}_{\theta} \dot{\bar{\xi}}_{\theta}+\frac{1}{\Gamma_{\lambda_{A}} \lambda_{A}} \widetilde{\lambda}_{A} \dot{\hat{\lambda}}_{A} .
\end{aligned}
$$

By using Lemma 1, it can be checked that

$$
\Lambda(\theta)\left[\varepsilon_{\theta}+d_{\theta}(t)\right] \leq\|\Lambda(\theta)\| \xi_{\theta} \leq \frac{\xi_{\theta} \Lambda(\theta) \Lambda^{T}(\theta)}{\sqrt{\Lambda(\theta) \Lambda^{T}(\theta)+\varepsilon_{\xi, \Lambda}^{2}}}+\xi_{\theta} \varepsilon_{\xi, \Lambda},
$$

where $\varepsilon_{\xi, \Sigma}>0$. Substituting (25) into (24), we can obtain that

$$
\begin{aligned}
\dot{V}_{1} \leq & -k_{1} s_{\theta}^{T} s_{\theta}{ }^{2 l-1}+\Lambda(\theta)\left(\begin{array}{c}
A_{\theta} \Omega_{\theta} e_{2}+A_{\theta} \Omega_{\theta} \omega_{v}-A_{\theta} \omega_{v c} \\
-\widetilde{O}_{t, \theta} \tanh \left(C_{t, \theta}\right)
\end{array}\right) \\
& -\frac{\widetilde{\xi}_{\theta} \Lambda(\theta) \Lambda^{T}(\theta)}{\sqrt{\Lambda(\theta) \Lambda^{T}(\theta)+\varepsilon_{\xi, \Lambda}^{2}}}+\xi_{\theta} \varepsilon_{\xi, \Lambda} \\
& +\frac{1}{\Gamma_{O_{t, \theta}}} \widetilde{O}_{t, \theta}^{T} \dot{\hat{\mathrm{O}}}_{t, \theta}+\frac{1}{\Gamma_{\xi_{\theta}}} \tilde{\xi}_{\theta} \dot{\vec{\xi}}_{\theta}+\frac{1}{\Gamma_{\lambda_{A}} \lambda_{A}} \widetilde{\lambda}_{A} \dot{\hat{\lambda}}_{A} .
\end{aligned}
$$

$$
\begin{aligned}
\Lambda(\theta) A_{\theta} \Omega_{\theta} \omega_{v} & =\Upsilon(\theta) \Omega_{\theta} \omega_{v}=-\Omega_{\theta} \frac{\left(\Upsilon(\theta) \hat{\lambda}_{A} \omega_{v c}\right)^{2}}{\sqrt{\left(\Upsilon(\theta) \hat{\lambda}_{A} \omega_{v c}\right)^{2}+\varepsilon_{A}^{2}}} \\
& \leq-\frac{\left(\Upsilon(\theta) \widehat{\lambda}_{A} \omega_{v c}\right)^{2}}{\lambda_{A} \sqrt{\left(\Upsilon(\theta) \hat{\lambda}_{A} \omega_{v c}\right)^{2}+\varepsilon_{A}^{2}}} \\
& \leq \frac{1}{\lambda_{A}}\left(\Upsilon(\theta) \hat{\lambda}_{A} \omega_{v c}+\varepsilon_{A}\right) .
\end{aligned}
$$

Therefore,

$$
\Lambda(\theta) A_{\theta} \Omega_{\theta} \omega_{v}-\Lambda(\theta) A_{\theta} \omega_{v c} \leq \frac{\tilde{\lambda}_{A}}{\lambda_{A}} \Upsilon(\theta) \omega_{v c}+\frac{\varepsilon_{A}}{\lambda_{A}} .
$$

Obviously,

$\Lambda(\theta) A \Omega_{\theta} e_{2}=\Upsilon(\theta) \Omega_{\theta} e_{2} \leq \lambda_{\max ^{2}}\left(\Omega_{\theta}\right)+\frac{1}{2} \Upsilon(\theta) \Upsilon^{T}(\theta) e_{2}^{T} e_{2}$.

Combining (26), (28), and (29), it can be proven that

$$
\begin{aligned}
\dot{V}_{1} \leq & -k_{1} s_{\theta}^{T} s_{\theta}^{2 l-1}-\Lambda(\theta) \widetilde{O}_{t, \theta} \tanh \left(C_{t, \theta}\right)-\widetilde{\xi}_{\theta} \Lambda(\theta) \varphi(\theta) \\
& +\lambda_{\max ^{2}}\left(\Omega_{\theta}\right)+\frac{1}{2} \Upsilon(\theta) \Upsilon^{T}(\theta) e_{2}^{T} e_{2}+\frac{\widetilde{\lambda}_{A}}{\lambda_{A}} \Upsilon(\theta) \omega_{v c} \\
& +\frac{1}{\Gamma_{O_{t, \theta}}} \widetilde{O}_{t, \theta}^{T} \dot{\hat{O}}_{t, \theta}+\frac{1}{\Gamma_{\xi_{\theta}}} \widetilde{\xi}_{\theta} \dot{\bar{\xi}}_{\theta}+\frac{1}{\Gamma_{\lambda_{A}} \lambda_{A}} \widetilde{\lambda}_{A} \dot{\hat{\lambda}}_{A}+\frac{\varepsilon_{A}}{\lambda_{A}}+\xi_{\theta} \varepsilon_{\xi, \Lambda} .
\end{aligned}
$$
$\widehat{\lambda}_{A}$ as

Considering (30), we design the adaptive laws for $\widehat{O}_{t, \theta}, \widehat{\xi}_{\theta}$,

$$
\begin{aligned}
\dot{\hat{O}}_{t, \theta} & =\Gamma_{O_{t, \theta}}\left(\Lambda^{T}(\theta) \circ \tanh \left(C_{t, \theta}\right)-\sigma_{O_{t, \theta}} \widehat{O}_{t, \theta}\right), \\
\dot{\vec{\xi}}_{\theta} & =\Gamma_{\xi_{\theta}}\left(\Lambda(\theta) \varphi(\theta)-\sigma_{\xi_{\theta}} \widehat{\xi}_{\theta}\right), \\
\dot{\hat{\lambda}}_{A} & =\Gamma_{\lambda_{A}}\left(-\Upsilon(\theta) \omega_{v c}-\sigma_{\lambda_{A}} \hat{\lambda}_{A}\right),
\end{aligned}
$$

where $\sigma_{O_{t \theta}}, \sigma_{\xi_{\theta}}, \sigma_{\lambda_{A}}>0$. Therefore, by using the update law (31), we know that

$$
\begin{aligned}
\dot{V}_{1} \leq & -k_{1} s_{\theta}^{T} s_{\theta}^{2 l-1}+\lambda_{\max ^{2}}\left(\Omega_{\theta}\right)+\frac{1}{2} \Upsilon(\theta) \Upsilon^{T}(\theta) e_{2}^{T} e_{2} \\
& -\sigma_{O_{t, \theta}} \widetilde{O}_{t, \theta}^{T} \widehat{O}_{t, \theta}-\sigma_{\xi_{\theta}} \widetilde{\xi}_{\theta} \widehat{\xi}_{\theta}-\frac{\sigma_{\lambda_{A}} \widetilde{\lambda}_{A} \widehat{\lambda}_{A}}{\lambda_{A}}+\frac{\varepsilon_{A}}{\lambda_{A}}+\xi_{\theta} \varepsilon_{\xi, \Lambda} .
\end{aligned}
$$

Since 


$$
\begin{aligned}
&-\sigma_{O_{t, \theta}} \widetilde{O}_{t, \theta}^{T} \widehat{O}_{t, \theta} \leq-\frac{1}{2} \sigma_{O_{t, \theta}}\left\|\widetilde{O}_{t, \theta}\right\|_{F}^{2}+\frac{1}{2} \sigma_{O_{t, \theta}}\left\|O_{t, \theta}\right\|_{F}^{2} \\
&-\sigma_{\xi} \widetilde{\xi}_{\theta} \widehat{\xi}_{\theta} \leq-\frac{1}{2} \sigma_{\xi_{\theta}} \widetilde{\xi}_{\theta}^{2}+\frac{1}{2} \sigma_{\xi_{\theta}} \xi_{\theta}^{2} \\
&-\frac{1}{\lambda_{A}} \sigma_{\lambda_{A}} \tilde{\lambda}_{A} \hat{\lambda}_{A} \leq-\frac{1}{2 \lambda_{A}} \sigma_{\lambda_{A}} \tilde{\lambda}_{A}^{2}+\frac{1}{2 \lambda_{A}} \sigma_{\lambda_{A}} \lambda_{A}^{2},
\end{aligned}
$$

substituting (33) into (32), we can rewrite (32) as

$$
\begin{aligned}
\dot{V}_{1} \leq & -k_{1}\left\|s_{\theta}\right\|^{2 l}+\frac{1}{2} \Upsilon(\theta) \Upsilon^{T}(\theta) e_{2}^{T} e_{2} \\
& -\frac{1}{2} \sigma_{O_{t, \theta}}\left\|\widetilde{O}_{t, \theta}\right\|_{F}^{2}-\frac{1}{2} \sigma_{\xi_{\theta}} \widetilde{\xi}_{\theta}^{2}-\frac{1}{2 \lambda_{A}} \sigma_{\lambda_{A}} \widetilde{\lambda}_{A}^{2} \\
& +\lambda_{\max ^{2}}\left(\Omega_{\theta}\right)+\frac{1}{2} \sigma_{O_{t, \theta}}\left\|O_{t, \theta}\right\|_{F}^{2}+\frac{1}{2} \sigma_{\xi_{\theta}} \xi_{\theta}^{2}+\frac{1}{2 \lambda_{A}} \sigma_{\lambda_{A}} \lambda_{A}^{2}+\frac{\varepsilon_{A}}{\lambda_{A}}+\xi_{\theta} \varepsilon_{\xi, \Lambda}
\end{aligned}
$$

According to Lemma 3, the following inequality holds:

$$
\begin{gathered}
\left(\frac{1}{2} \sigma_{O_{t, \theta}}\left\|\widetilde{O}_{t, \theta}\right\|_{F}^{2}\right)^{l} \leq \iota(1-l)+\frac{1}{2} \sigma_{O_{t, \theta}}\left\|\widetilde{O}_{t, \theta}\right\|_{F}^{2}, \\
\left(\frac{1}{2} \sigma_{\xi_{\theta}} \widetilde{\xi}_{\theta}^{2}\right)^{l} \leq \iota(1-l)+\frac{1}{2} \sigma_{\xi_{\theta}} \widetilde{\xi}_{\theta}^{2}, \\
\left(\frac{1}{2 \lambda_{A}} \sigma_{\lambda_{A}} \widetilde{\lambda}_{A}^{2}\right)^{l} \leq \iota(1-l)+\frac{1}{2 \lambda_{A}} \sigma_{\lambda_{A}} \widetilde{\lambda}_{A}^{2} .
\end{gathered}
$$

Therefore, we can get that

$$
\begin{aligned}
\dot{V}_{1} \leq & -\lambda_{\min }\left(k_{1}\right)\left\|s_{\theta}\right\|^{2 l}+\frac{1}{2} \Upsilon(\theta) \Upsilon^{T}(\theta) e_{2}^{T} e_{2} \\
& -\left(\frac{1}{2} \sigma_{O_{t, \theta}}\left\|\widetilde{O}_{t, \theta}\right\|_{F}^{2}\right)^{l}-\left(\frac{1}{2} \sigma_{\xi_{\theta}} \widetilde{\xi}_{\theta}^{2}\right)^{l}-\left(\frac{1}{2 \lambda_{A}} \sigma_{\lambda_{A}} \widetilde{\lambda}_{A}^{2}\right)^{l}+\varepsilon_{\Upsilon, 1},
\end{aligned}
$$

where

$$
\begin{aligned}
\mathcal{E}_{\Upsilon, 1}= & \frac{1}{2} \sigma_{O_{t, \theta}}\left\|O_{t, \theta}\right\|_{F}^{2}+\frac{1}{2} \sigma_{\xi_{\theta}} \xi_{\theta}^{2}+\frac{1}{2 \lambda_{A}} \sigma_{\lambda_{A}} \lambda_{A}^{2}+\lambda_{\max ^{2}}\left(\Omega_{\theta}\right) \\
& +3 \iota(1-l)+\frac{\varepsilon_{A}}{\lambda_{A}}+\xi_{\theta} \varepsilon_{\xi, \Lambda} .
\end{aligned}
$$

3.2. BLSTM-Based Input Uncertainty Suppressed Outer Loop Finite-Time Controller Design. According to (7), we can get that

$$
\dot{e}_{2}=\left[B_{\omega}+\Delta B_{\omega}\right] \delta+C(\omega)+f(\theta, \omega)+d_{\omega}(t)-\dot{\omega}_{v} .
$$

A BLSTM network $O_{t, \omega} \tanh \left(C_{t, \omega}\right)$ is utilized to handle $f(\theta, \omega)$. Define the BLSTM node number in the outer loop as $N_{2}$. We can also design the self-adjust strategy for the nodes number as

$$
\begin{cases}N_{2}=N_{2}+n_{2 \text { ad }}, & \int_{t_{2}-1}^{t_{2}}\left\|e_{2}(\tau)\right\| \mathrm{d} \tau>\chi_{2 \text { up }}, \\ N_{2}=N_{2}, & \chi_{2 \text { low }} \leq \int_{t_{2}-1}^{t_{2}}\left\|e_{2}(\tau)\right\| \mathrm{d} \tau \leq \chi_{2 \text { up }}, \\ N_{2}=N_{2}-n_{2 \text { ad }}, & \int_{t_{2}-1}^{t_{2}}\left\|e_{2}(\tau)\right\| \mathrm{d} \tau<\chi_{2 \text { low }},\end{cases}
$$

where $\varepsilon_{\omega}$ represents the adjustment and it is a designed positive integer; $\chi_{2 \text { low }}, \chi_{2 \text { up }}$ are designed positive constants, denoting the adjustment threshold; and $t_{2}$ represents the time series to complete the judgment and adjustment.

According to (38), we design

$$
\begin{aligned}
\delta_{v c}= & B_{\omega}^{-1}\left(-k_{2}\left\|e_{2}\right\|^{2 l-1}-\frac{1}{2} e_{2} \Upsilon(\theta) \Upsilon^{T}(\theta)\right. \\
& \left.-C(\omega)-\widehat{O}_{t, \omega} \tanh \left(C_{t, \omega}\right)-\widehat{\xi}_{\omega} \varphi\left(e_{2}\right)+\dot{\omega}_{v}\right),
\end{aligned}
$$

$$
\varphi\left(e_{2}\right)=\frac{e_{2}}{\sqrt{e_{2}^{T} e_{2}+\varepsilon_{\xi, e_{2}}^{2}}}, \quad \forall \varepsilon_{\xi, e_{2}}>0,
$$

where $\delta_{v c}$ is the virtual control signal of $\delta, k_{2}$ is the control gain, $B_{\omega}^{-1}=B_{\omega}^{T} / B_{\omega} B_{\omega}^{T}$, and $\widehat{\xi}_{\omega}$ represents the estimations of $\xi_{\omega}$ $\left(\xi_{\omega}=\sup _{t \geq 0}\left\|\varepsilon_{\omega}+d_{\omega}(t)\right\|\right)$.

To deal with the perturbations of $B_{\omega}$, we define $\lambda_{B}=1 / \inf \left(\lambda_{\min }\left(\Omega_{\omega}\right)\right)$. Accordingly, we design

$$
\delta_{v}=-\frac{\widehat{\lambda}_{B} \delta_{v c}\left(e_{2}^{T} B_{\omega} \hat{\lambda}_{B} \delta_{v c}\right)}{\sqrt{\left(e_{2}^{T} B_{\omega} \hat{\lambda}_{B} \delta_{v c}\right)^{2}+\varepsilon_{B}^{2}}}, \quad \forall \varepsilon_{B}>0
$$

where $\hat{\lambda}_{B}$ is the estimation of $\lambda_{B}$. Substituting (40) and (41) into (38) yields

$$
\begin{aligned}
\dot{e}_{2}= & -\frac{1}{2} e_{2} \Upsilon(\theta) \Upsilon^{T}(\theta)-k_{2}\left\|e_{2}\right\|^{2 l-1}+B_{\omega} \Omega_{\omega} \delta-B_{\omega} \delta_{v c} \\
& -\widetilde{O}_{t, \omega} \tanh \left(C_{t, \omega}\right)-\widehat{\xi}_{\omega} \varphi\left(e_{2}\right)+d_{\omega}(t)+\varepsilon_{\omega} .
\end{aligned}
$$

Select the following Lyapunov function:

$$
V_{2}=\frac{1}{2} e_{2}^{T} e_{2}+\frac{1}{2 \Gamma_{O_{t, \omega}}} \widetilde{O}_{t, \omega}^{T} \widetilde{O}_{t, \omega}+\frac{1}{2 \Gamma_{\xi_{\omega}}} \widetilde{\xi}_{\omega}^{2}+\frac{1}{2 \Gamma_{\lambda_{B}} \lambda_{B}} \widetilde{\lambda}_{B}^{2},
$$

where $\widetilde{O}_{t, \omega}=\widehat{O}_{t, \omega}-O_{t, \omega}, \widetilde{\xi}_{\omega}=\widehat{\xi}_{\omega}-\xi_{\omega}, \tilde{\lambda}_{B}=\widehat{\lambda}_{B}-\lambda_{B}, \Gamma_{O_{t, \omega}}$, $\Gamma_{\xi_{\omega}}, \Gamma_{\lambda_{B}}>0$. We can take the differential of $V_{2}$ as

$$
\begin{aligned}
\dot{V}_{2}= & e_{2}^{T} \dot{e}_{2}+\frac{1}{\Gamma_{O_{t, \omega}}} \widetilde{O}_{t, \omega}^{T} \dot{\hat{O}}_{t, \omega}+\frac{1}{\Gamma_{\xi}} \widetilde{\xi}_{\omega} \dot{\hat{\xi}}_{\omega}+\frac{1}{\Gamma_{\lambda_{B}} \lambda_{B}} \widetilde{\lambda}_{B} \dot{\hat{\lambda}}_{B} \\
= & e_{2}^{T}\left(\begin{array}{c}
B_{\omega} \Omega_{\omega} \delta-B_{\omega} \delta_{v c}-\frac{1}{2} e_{2} \Upsilon(\theta) \Upsilon^{T}(\theta)-k_{2} e_{2}{ }^{2 l-1} \\
-\widetilde{O}_{t, \omega} \tanh \left(C_{t, \omega}\right)-\widehat{\xi}_{\omega} \varphi\left(e_{2}\right)+\varepsilon_{\omega}+d_{\omega}(t)
\end{array}\right) \\
& +\frac{1}{\Gamma_{O_{t, \omega}}} \widetilde{O}_{t, \omega}^{T} \dot{\hat{O}}_{t, \omega}+\frac{1}{\Gamma_{\xi_{\omega}}} \widetilde{\xi}_{\omega} \dot{\hat{\xi}}_{\omega}+\frac{1}{\Gamma_{\lambda_{B}} \lambda_{B}} \widetilde{\lambda}_{B} \dot{\hat{\lambda}}_{B} .
\end{aligned}
$$


By using Lemma 1, we know that

$$
e_{2}^{T}\left[\varepsilon_{\omega}+d_{\omega}(t)\right] \leq \xi_{\omega}\left\|e_{2}\right\| \leq \xi_{\omega} e_{2}^{T} \varphi\left(e_{2}\right)+\xi_{\omega} \varepsilon_{\xi, e_{2}} .
$$

Then, from (41) and Lemma 1, we can obtain that

$$
\begin{aligned}
e_{2}^{T} B_{\omega} \Omega_{\omega} \delta_{v} & =-\Omega_{\omega} \frac{e_{2}^{T} B_{\omega} \hat{\lambda}_{B} \delta_{v c}\left(e_{2}^{T} B_{\omega} \hat{\lambda}_{B} \delta_{v c}\right)}{\sqrt{\left(e_{2}^{T} B_{\omega} \hat{\lambda}_{B} \delta_{v c}\right)^{2}+\varepsilon_{B}^{2}}} \\
& \leq-\frac{1}{\lambda_{B}}\left(\left\|e_{2}^{T} B_{\omega} \hat{\lambda}_{B} \delta_{v c}\right\|-\varepsilon_{B}\right) \\
& \leq \frac{\hat{\lambda}_{B}}{\lambda_{B}} e_{2}^{T} B_{\omega} \delta_{v c}+\frac{\varepsilon_{B}}{\lambda_{B}} .
\end{aligned}
$$

Therefore,

$$
e_{2}^{T} B_{\omega} \Omega_{\omega} \delta-e_{2}^{T} B_{\omega} \delta_{v c} \leq \frac{\tilde{\lambda}_{B}}{\lambda_{B}} e_{2}^{T} B_{\omega} \delta_{v c}+\frac{\varepsilon_{B}}{\lambda_{B}} .
$$
that

By substituting (45) and (47) into (44), it can be obtained

$$
\begin{aligned}
\dot{V}_{2} \leq & -k_{2}\left\|e_{2}\right\|^{2 l}-\frac{1}{2} e_{2}^{T} e_{2} \Upsilon(\theta) \Upsilon^{T}(\theta)+\frac{\tilde{\lambda}_{B}}{\lambda_{B}} e_{2}^{T} B_{\omega} \delta_{v c} \\
& +\frac{\varepsilon_{B}}{\lambda_{B}}-e_{2}^{T} \widetilde{O}_{t, \omega} \tanh \left(C_{t, \omega}\right) \\
& -\widetilde{\xi}_{\omega} e_{2}^{T} \varphi\left(e_{2}\right)+\xi_{\omega} \varepsilon_{\xi, e_{2}}+\frac{1}{\Gamma_{O_{t, \omega}}} \widetilde{O}_{t, \omega}^{T} \dot{\hat{O}}_{t, \omega} \\
& +\frac{1}{\Gamma_{\xi_{\omega}}} \widetilde{\xi}_{\omega} \dot{\vec{\xi}}_{\omega}+\frac{1}{\Gamma_{\lambda_{B}} \lambda_{B}} \widetilde{\lambda}_{B} \dot{\hat{\lambda}}_{B} .
\end{aligned}
$$

In view of (41), the adaptive laws for $\widehat{O}_{t, \omega}, \widehat{\xi}_{\omega}, \widehat{\lambda}_{B}$ are designed as

$$
\begin{aligned}
\dot{\widehat{O}}_{t, \omega} & =\Gamma_{O_{t, \omega}}\left(e_{2} \circ \tanh \left(C_{t, \omega}\right)-\sigma_{O_{t, \omega}} \widehat{O}_{t, \omega}\right), \\
\dot{\vec{\xi}}_{\omega} & =\Gamma_{\xi_{\omega}}\left(e_{2}^{T} \varphi\left(e_{2}\right)-\sigma_{\xi_{\omega}} \widehat{\xi}_{\omega}\right), \\
\dot{\hat{\lambda}}_{B} & =-\Gamma_{\lambda_{B}}\left(e_{2}^{T} B_{\omega} \delta_{v c}-\sigma_{\lambda_{B}} \widehat{\lambda}_{B}\right),
\end{aligned}
$$

where $\sigma_{O_{t, \omega}}, \sigma_{\xi_{\omega}}, \sigma_{\lambda_{B}}>0$. Combining (48) with (49) generates

$$
\begin{aligned}
\dot{V}_{2} \leq & -k_{2}\left\|e_{2}\right\|^{2 l}-\frac{1}{2} e_{2}^{T} e_{2} \Upsilon(\theta) \Upsilon^{T}(\theta)-\sigma_{O_{t, \omega}} \widetilde{O}_{t, \omega}^{T} \widehat{O}_{t, \omega} \\
& -\sigma_{\xi_{\omega}} \widetilde{\xi}_{\omega} \widehat{\xi}_{\omega}-\frac{\sigma_{\lambda_{B}} \widetilde{\lambda}_{B} \widehat{\lambda}_{B}}{\lambda_{B}}+\xi_{\omega} \varepsilon_{\xi, e_{2}}+\frac{\varepsilon_{B}}{\lambda_{B}} .
\end{aligned}
$$

Therefore,

$$
\begin{aligned}
\dot{V}_{2} \leq & -\frac{1}{2} e_{2}^{T} e_{2} \Upsilon(\theta) \Upsilon^{T}(\theta)-\lambda_{\min }\left(k_{2}\right)\left\|e_{2}\right\|^{2 l} \\
& -\frac{1}{2} \sigma_{O_{t, \omega}}\left\|\widetilde{O}_{t, \omega}\right\|_{F}^{2}+\frac{1}{2} \sigma_{O_{t, \omega}}\left\|O_{t, \omega}\right\|_{F}^{2} \\
& -\frac{1}{2} \sigma_{\xi_{\omega}} \widetilde{\xi}_{\omega}^{2}+\frac{1}{2} \sigma_{\xi_{\omega}} \xi_{\omega}^{2}-\frac{1}{2 \lambda_{B}} \sigma_{\lambda_{B}} \widetilde{\lambda}_{B}^{2}+\frac{1}{2 \lambda_{B}} \sigma_{\lambda_{B}} \lambda_{B}^{2}+\xi_{\omega} \varepsilon_{\xi, e_{2}}+\frac{\varepsilon_{B}}{\lambda_{B}} .
\end{aligned}
$$

Next, by applying Lemma 3, we can obtain

$$
\begin{gathered}
\left(\frac{1}{2} \sigma_{O_{t, \omega}}\left\|\widetilde{O}_{t, \omega}\right\|_{F}^{2}\right)^{l} \leq(1-l) \iota+\frac{1}{2} \sigma_{O_{t, \omega}}\left\|\widetilde{O}_{t, \omega}\right\|_{F}^{2}, \\
\left(\frac{1}{2} \sigma_{\xi_{\omega}} \widetilde{\xi}_{\omega}^{2}\right)^{l} \leq(1-l) \iota+\frac{1}{2} \sigma_{\xi_{\omega}} \widetilde{\xi}_{\omega}^{2}, \\
\left(\frac{1}{2 \lambda_{B}} \sigma_{\lambda_{B}} \tilde{\lambda}_{B}^{2}\right)^{l} \leq(1-l) \iota+\frac{1}{2 \lambda_{B}} \sigma_{\lambda_{B}} \widetilde{\lambda}_{B}^{2} .
\end{gathered}
$$

Hence, we can get that

$$
\begin{aligned}
\dot{V}_{2} \leq & -\frac{1}{2} e_{2}^{T} e_{2} \Upsilon(\theta) \Upsilon^{T}(\theta)-\lambda_{\min }\left(k_{2}\right)\left\|e_{2}\right\|^{2 l} \\
& -\left(\frac{1}{2} \sigma_{O_{t, \omega}}\left\|\widetilde{O}_{t, \omega}\right\|_{F}^{2}\right)^{l}-\left(\frac{1}{2} \sigma_{\xi_{\omega}} \widetilde{\xi}_{\omega}^{2}\right)^{l}-\left(\frac{1}{2 \lambda_{B}} \sigma_{\lambda_{B}} \widetilde{\lambda}_{B}^{2}\right)^{l}+\varepsilon_{\Upsilon, 2},
\end{aligned}
$$

where

$$
\begin{aligned}
\varepsilon_{\Upsilon, 2}= & \frac{1}{2} \sigma_{O_{t, \omega}}\left\|O_{t, \omega}\right\|_{F}^{2}+\frac{1}{2} \sigma_{\xi_{\omega}} \xi_{\omega}^{2}+\frac{1}{2 \lambda_{B}} \sigma_{\lambda_{B}} \lambda_{B}^{2} \\
& +\xi_{\omega} \varepsilon_{\xi, e_{2}}+\frac{\varepsilon_{B}}{\lambda_{B}}+3(1-l) l .
\end{aligned}
$$

3.3. Stability Analysis of the Proposed BLSTM-Based FiniteTime Control Law. The main result on global adaptive tracking is stated in this section.

Theorem 1. Consider the closed-loop system consisting of the AUS (7), the indirect virtual controllers (19) and (40), the final virtual controllers (20) and (41), and the adaptive update laws (31) and (49) and suppose that Assumptions 1 and 2 are satisfied. Moreover, it is guaranteed that the nonlinear mapping error $s_{\theta}$ is bounded and can converge in finite time, and thus the tracking error can converge to a small compact set in finite time in the presence of the uncertainties and the faulty elevator outputs.

Proof. According to (36) and (53), we define $V=V_{1}+V_{2}$. Thus,

$$
\begin{aligned}
\dot{V}= & \dot{V}_{1}+\dot{V}_{2} \\
\leq & -\lambda_{\min }\left(k_{1}\right)\left(s_{\theta}^{T} s_{\theta}\right)^{2 l}-\lambda_{\min }\left(k_{2}\right)\left\|e_{2}\right\|^{2 l} \\
& -\left(\frac{1}{2} \sigma_{O_{t, \theta}}\left\|\widetilde{O}_{t, \theta}\right\|_{F}^{2}\right)^{l}-\left(\frac{1}{2} \sigma_{\xi_{\theta}} \widetilde{\xi}_{\theta}^{2}\right)^{l}-\left(\frac{1}{2 \lambda_{A}} \sigma_{\lambda_{A}} \widetilde{\lambda}_{A}^{2}\right)^{l} \\
& -\left(\frac{1}{2} \sigma_{O_{t, \omega}}\left\|\widetilde{O}_{t, \omega}\right\|_{F}^{2}\right)^{l}-\left(\frac{1}{2} \sigma_{\xi_{\omega}} \widetilde{\xi}_{\omega}^{2}\right)^{l}-\left(\frac{1}{2 \lambda_{B}} \sigma_{\lambda_{B}} \widetilde{\lambda}_{B}^{2}\right)^{l}+\varepsilon_{\Upsilon 1}+\varepsilon_{\Upsilon 2} .
\end{aligned}
$$

Define 
TABle 1: The parameters of the AUS.

\begin{tabular}{lcc}
\hline$V=10 \mathrm{~m} / \mathrm{s}$ & $m=28 \mathrm{~kg}$ & $b=3.1 \mathrm{~m}$ \\
$c=0.58 \mathrm{~m}$ & $S=1.8 \mathrm{~m}^{2}$ & $\rho=1.29 \mathrm{~kg} / \mathrm{m}^{3}$ \\
$I_{x x}=2.56 \mathrm{~kg} \cdot \mathrm{m}^{2}$ & $I_{z x}=0.5 \mathrm{~kg} \cdot \mathrm{m}^{2}$ & $I_{z z}=11.3 \mathrm{~kg} \cdot \mathrm{m}^{2}$ \\
$I_{y y}=10.9 \mathrm{~kg} \cdot \mathrm{m}^{2}$ & $I_{x z}=0.5 \mathrm{~kg} \cdot \mathrm{m}^{2}$ & \\
\hline
\end{tabular}

TABLE 2: The uncertainties of the four cases.

\begin{tabular}{l}
\hline$f(\theta) \quad\left[\begin{array}{c}0.002+0.3 \times \sin (6.28 t / 10) \\
0.001+0.2 \times \\
\sin (6.28 t / 10) \\
0.002+0.01 \times \sin (6.28 t / 10)\end{array}\right]$ \\
\hline$f(\theta, \omega) \quad\left[\begin{array}{l}0.007+0.6 \times \sin (6.28 t / 10) \\
0.001+0.2 \times \sin (6.28 t / 10) \\
0.004+0.3 \times \sin (6.28 t / 10)\end{array}\right]$ \\
\hline
\end{tabular}

TABLE 3: The parameters of the system based on BLSTM networks.

\begin{tabular}{lr}
\hline The parameters based on BLSTM networks in the inner loop & $W_{i i}=0.1, W_{o i}=1, b_{i i}=2, b_{o i}=2, N_{\text {inner }}(0)=7$ \\
The parameters based on BLSTM networks in the outer loop & $W_{i o}=0.1, W_{o o}=1, b_{i o}=5, b_{o o}=12, N_{\text {outer }}(0)=10$
\end{tabular}

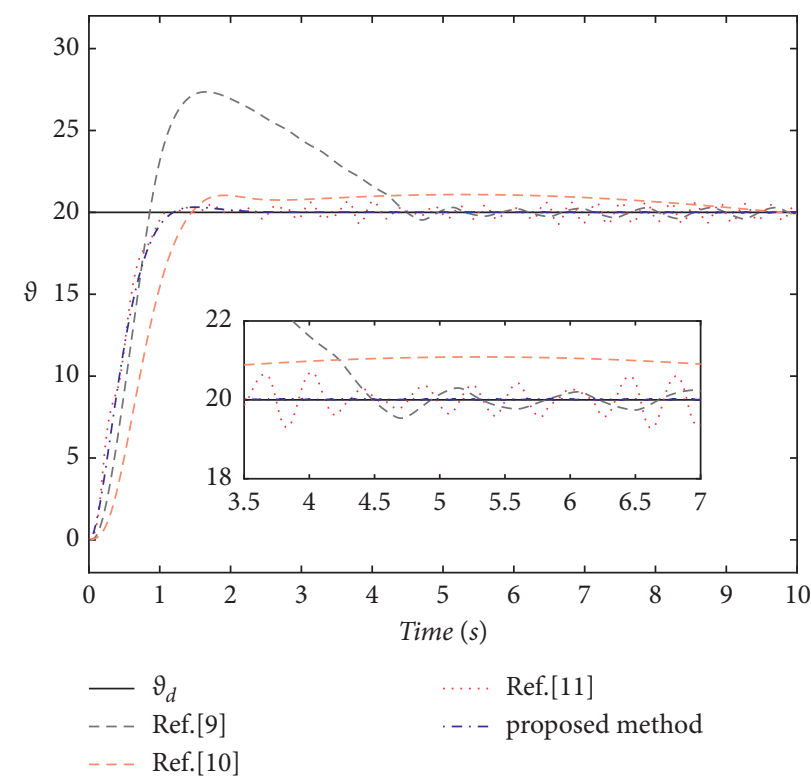

Figure 3: The performance of $\vartheta$ under four cases.

$$
\begin{aligned}
\sigma= & \min \left\{\begin{array}{c}
4 \lambda_{\min }\left(k_{1}\right), 2 \lambda_{\min }\left(k_{2}\right),\left(\sigma_{O_{t, \theta}} \Gamma_{O_{t, \theta}}\right)^{l},\left(\sigma_{\xi_{\theta}} \Gamma_{\xi_{\theta}}\right)^{l}, \\
\left(\sigma_{\lambda_{A}} \Gamma_{\lambda_{A}}\right)^{l},\left(\sigma_{O_{t, \omega}} \Gamma_{O_{t, \omega}}\right)^{l},\left(\sigma_{\xi_{\omega}} \Gamma_{\xi_{\omega}}\right)^{l},\left(\sigma_{\lambda_{B}} \Gamma_{\lambda_{B}}\right)^{l}
\end{array}\right\}, \\
\varepsilon_{\Upsilon}= & \varepsilon_{\Upsilon 1}+\varepsilon_{\Upsilon 2} \\
= & \frac{1}{2} \sigma_{O_{t, \theta}}\left\|O_{t, \theta}\right\|_{F}^{2}+\frac{1}{2} \sigma_{\xi_{\theta}} \xi_{\theta}^{2}+\frac{1}{2 \lambda_{A}} \sigma_{\lambda_{A}} \lambda_{A}^{2}+\lambda_{\max ^{2}}\left(\Omega_{\theta}\right)+\frac{\varepsilon_{A}}{\lambda_{A}}+\xi_{\theta} \varepsilon_{\xi, \Lambda} \\
& +\frac{1}{2} \sigma_{O_{t, \omega}}\left\|O_{t, \omega}\right\|_{F}^{2}+\frac{1}{2} \sigma_{\xi_{\omega}} \xi_{\omega}^{2}+\frac{1}{2 \lambda_{B}} \sigma_{\lambda_{B}} \lambda_{B}^{2}+\xi_{\omega} \varepsilon_{\xi, e_{2}}+\frac{\varepsilon_{B}}{\lambda_{B}}+6(1-l) l .
\end{aligned}
$$

We can define that

$$
\dot{V} \leq-\sigma V^{l}+\varepsilon_{\curlyvee}
$$

By solving (55), we can get that

$$
V(t) \leq\left[V^{1-l}(0)-\alpha \sigma(1-l) t\right]^{(1 /(1-l))}
$$

where $0<\alpha<1$. Meanwhile, let

$$
T=\frac{1}{\alpha \sigma(1-l)}\left\{V^{1-l}(0)-\left[\frac{\mathcal{E}_{\Upsilon}}{(1-\alpha) \sigma}\right]^{(1 /(1-l))}\right\},
$$

where $V(0)$ represents the initial value of $V(t)$. According to Lemma 2, for $t \geq T, V^{l} \leq \varepsilon_{\Upsilon} /(1-\alpha) \sigma$, that is, all the signals of the closed-loop system are SGPFS. 


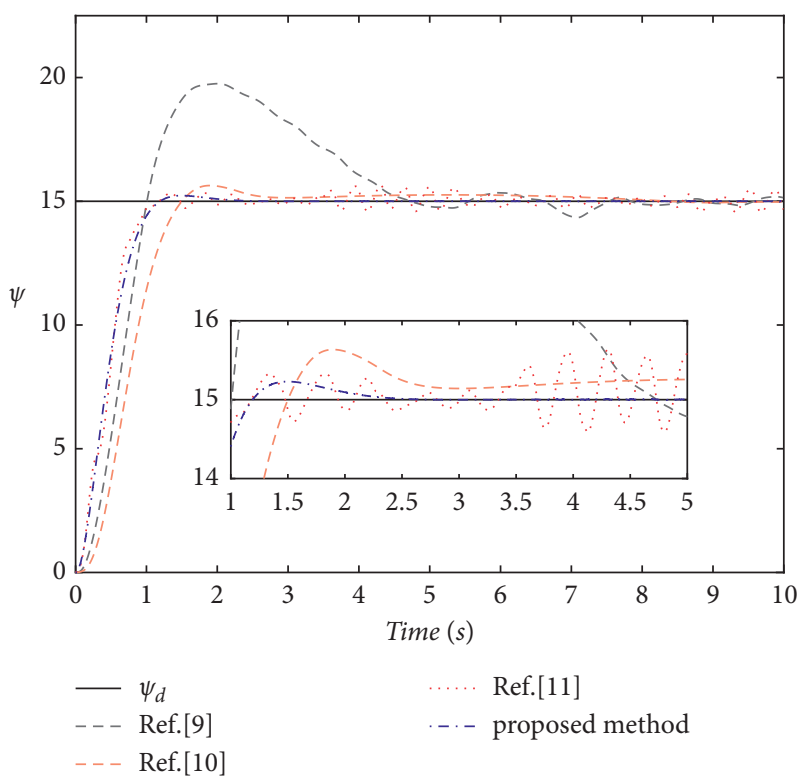

Figure 4: The performance of $\psi$ under four cases.

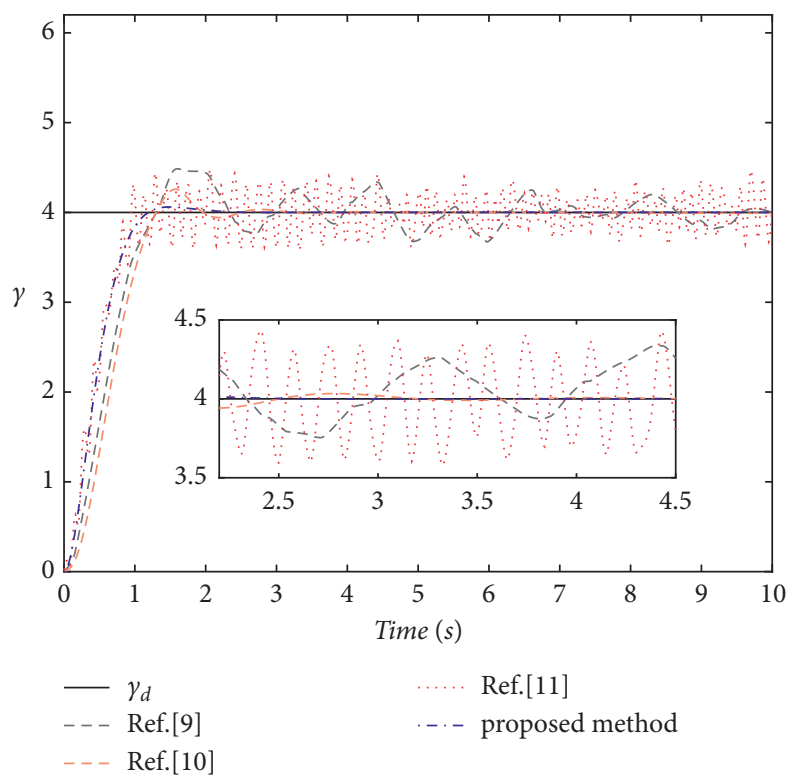

FIgURE 5: The performance of $\gamma$ under four cases.

Furthermore, according to the definition of $V$, it can be inferred that for $\forall t \geq T$,

$$
s_{\theta}^{T} s_{\theta} \leq 2\left(\frac{\mathcal{E}_{\Upsilon}}{(1-\alpha) \sigma}\right)^{(1 / 2 l)}
$$

holds. In other words, after the finite time $T, s_{\theta}$ remains in a small neighborhood of the origin. Thus, the tracking errors can converge to a desired compact set in a finite time and never violate the predefined constraint. The proof is completed.

\section{Simulation Study}

In this section, four cases of numerical examples, including the proposed control method, one without BLSTM, one without output constraint, and one without finite time, are carried out in the simulation.

In the simulation, the AUS flight height is $h=1000 \mathrm{~m}$, and the initial attitude angles and angular velocities are set as zero. The desired signals are selected as $\vartheta_{d}=20^{\circ}, \psi_{d}=15^{\circ}$, and $\gamma_{d}={ }^{\circ}$, and the simulation time is $T=10 \mathrm{~s}$. The parameters of AUS are provided in Table 1. 


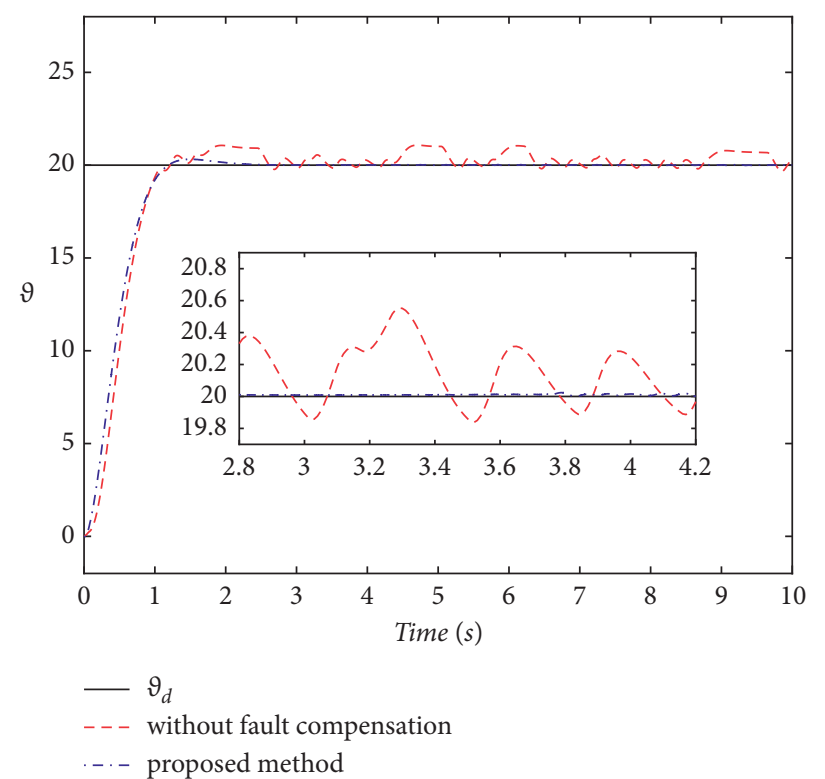

Figure 6: Trajectory of system state $\vartheta$ in the case of fault-tolerant controller $\delta_{v}$ and controller $\delta_{v c}$.

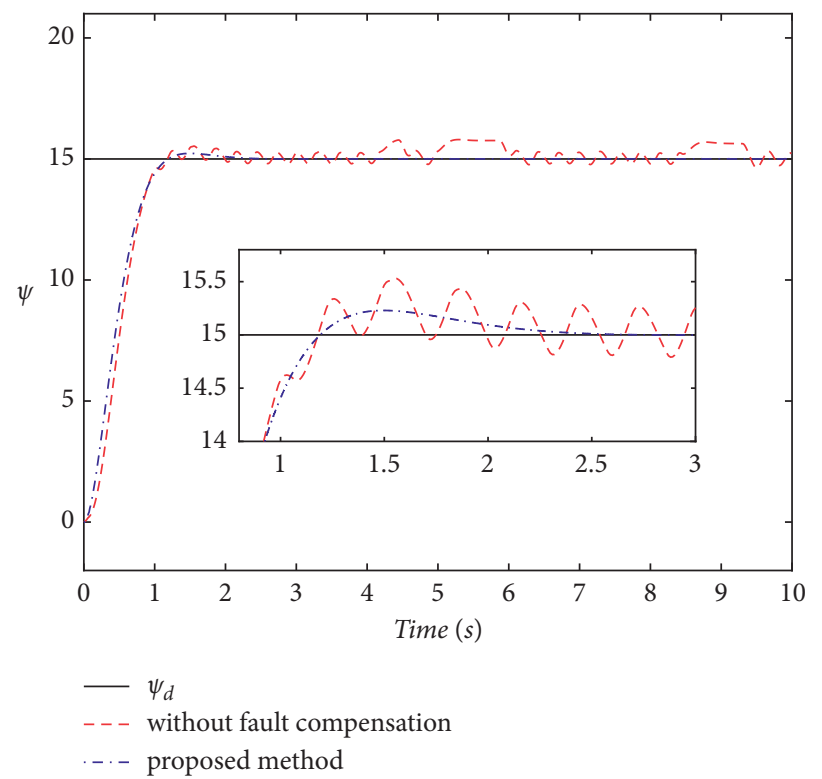

Figure 7: Trajectory of system state $\psi$ in the case of fault-tolerant controller $\delta_{v}$ and controller $\delta_{v c}$.

The uncertainties are chosen as $\xi_{\theta}=[0.006$, $0.002,0.003]^{T}$ and $\xi_{\omega}=[0.007,0.001,0.004]^{T}$. Simultaneously, the uncertainties of the four cases considered in the simulations are given in Table 2 . In this paper, the parameters of BLSTM network are given in Table 3.

The simulation results are given in Figures 3-11. Meanwhile, the control performances under four cases are shown in Figures 3-5, from which we can see that the attitude angles of the four methods are quiet different. In Case 4, we apply the proposed method according to the analysis in Section 4 , and it can track the desired signal closely in a finite time. However, for comparison, in Case 1, it shows that the proposed method can induce more overshoot without output constraint. Moreover, in Case 2, we apply the proposed control method with LSTM instead of BLSTM, which may induce tracking errors. That is to say, the BLSTM network can guarantee more accurate tracking accuracy. In Case 3, it is assumed that without finite time, the proposed method will converge in a longer time. Therefore, we know that the proposed BLSTM-based control law can achieve satisfactory control performance. From Figures 6-8, It can be seen that the proposed method with fault compensation has more advantages in control effect than the method without fault compensation and can effectively reduce the oscillation and realize stable control. The node number of BLSTM network is shown in Figure 11. Also, it can be seen that the nodes are variable with the tracking error, which will converge to a fixed value when the tracking error is stable. 


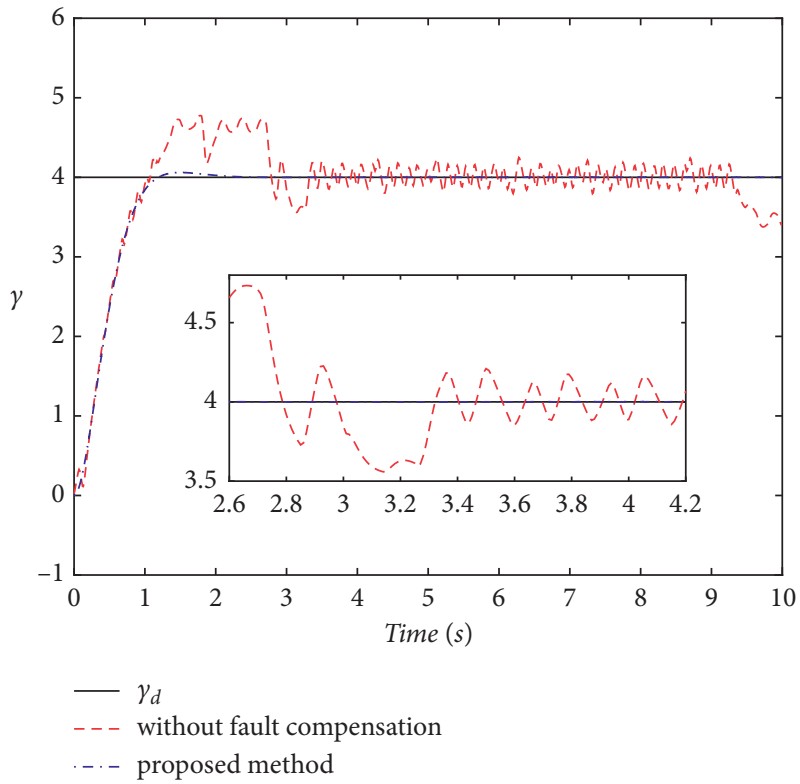

Figure 8: Trajectory of system state $\gamma$ in the case of fault-tolerant controller $\delta_{v}$ and controller $\delta_{v c}$.
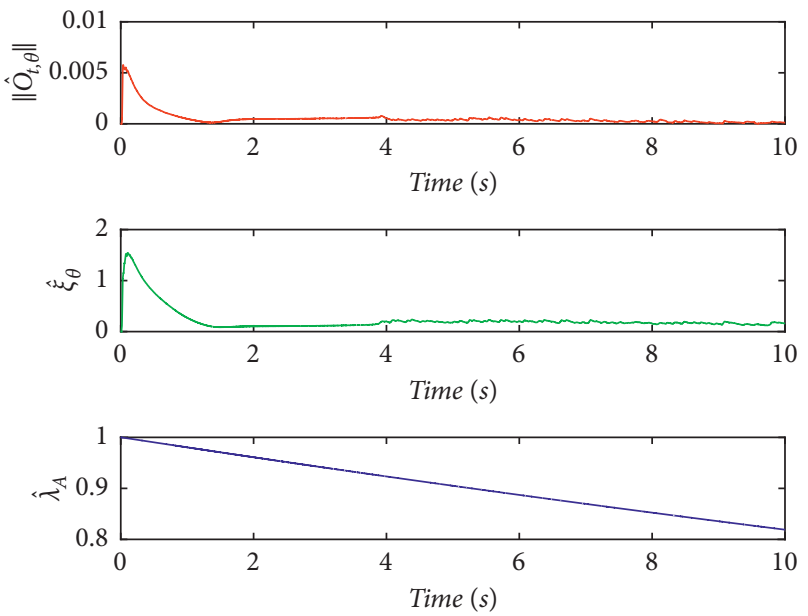

FIGURE 9: The trajectories of adaptive parameters in inner loop with the proposed method.
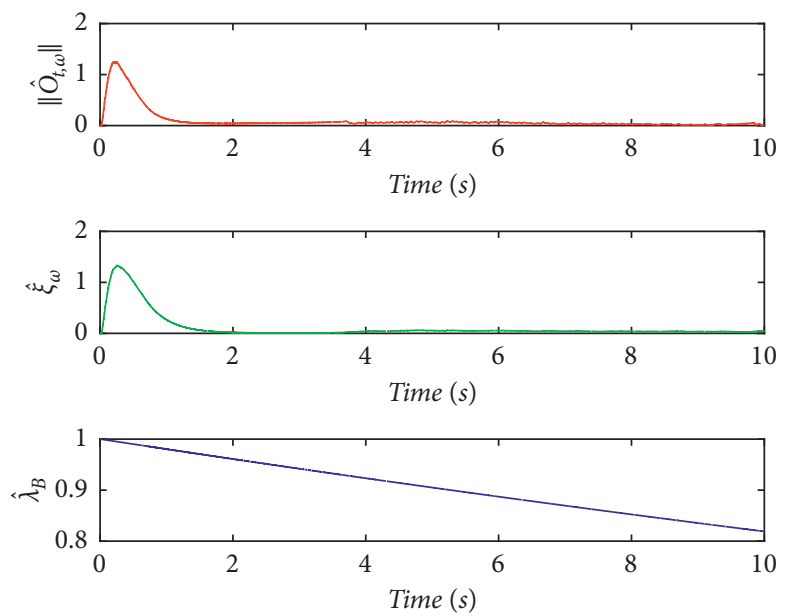

FIgURE 10: The trajectories of adaptive parameters in outer loop with the proposed method. 

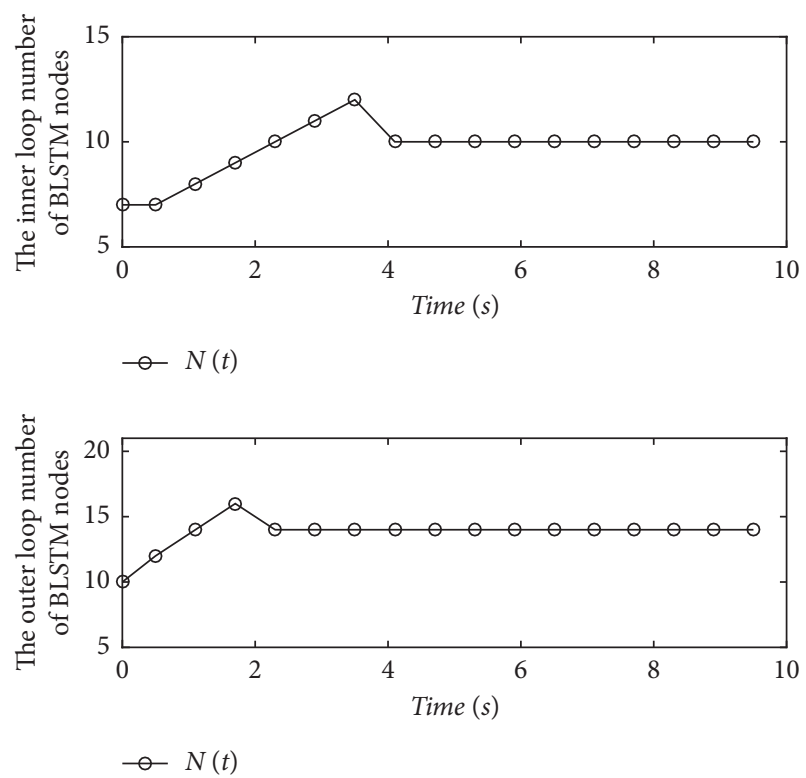

Figure 11: The trajectory of BLSTM node number.

Therefore, it can be concluded that the proposed BLSTMbased adaptive control algorithm can achieve favorable control results for the AUSs with multiple uncertainties.

\section{Conclusions}

In this paper, we propose a BLSTM-based adaptive finitetime control structure for a class of AUSs. The novel BLSTM which possesses both the memory function and rapid learning speed has been constructed. The fractionalorder control law and the corresponding adaptive laws are designed. Combining the BLSTM, the transformation of the tracking errors, and the adaptive finite-time control law, a novel BLSTM-based intelligent adaptive finite-time control structure has been established for the AUSs under output constraints. The simulation results demonstrate the effectiveness of the proposed BLSTM-based intelligent adaptive finite-time control method and reveal that the introduction of the BLSTM can improve the control performance for the AUSs. In future work, the BLSTMbased adaptive finite-time control method proposed in this paper will be used to deal with the AUSs with stochastic disturbances.

\section{Data Availability}

The data used to support the findings of this study are included within the article.

\section{Conflicts of Interest}

The authors declare that they have no conflicts of interest.

\section{Acknowledgments}

This study was supported in part by the National Natural Science Foundation of China under grant no. 11772256 and sponsored by the Innovation Foundation for Doctor Dissertation of Northwestern Polytechnical University (G2017KY0412).

\section{References}

[1] Y.-M. Li, X. Min, and S. Tong, "Adaptive fuzzy inverse optimal control for uncertain strict-feedback nonlinear systems," IEEE Transactions on Fuzzy Systems, vol. 28, no. 10, pp. 2363-2374, 2020.

[2] Y. Li, T. Yang, and S. Tong, "Adaptive neural networks finitetime optimal control for a class of nonlinear systems," IEEE Transactions on Neural Networks and Learning Systems, vol. 31, no. 11, pp. 4451-4460, 2020.

[3] D. Yu, C. L. P. Chen, C. E. Ren, and S. Sui, "Swarm control for self-organized system with fixed and switching topology," IEEE Transactions on Cybernetics, vol. 50, no. 99, pp. 44814494, 2020.

[4] Y.-J. Liu and S. Tong, "Barrier Lyapunov Functions-based adaptive control for a class of nonlinear pure-feedback systems with full state constraints," Automatica, vol. 64, pp. 70-75, 2016.

[5] D. Yu, C. L. P. Chen, and H. Xu, "Fuzzy swarm control based on sliding-mode strategy with self-organized omnidirectional mobile robots system," IEEE Transactions on Systems, Man, and Cybernetics: Systems, no. 99, pp. 1-13, 2021.

[6] S. Sui, C. L. P. Chen, and S. Tong, "Neural-network-based adaptive dsc design for switched fractional-order nonlinear systems," IEEE Transactions on Neural Networks and Learning Systems, no. 99, pp. 1-10, 2020.

[7] S. Sui, C. Chen, and S. Tong, "Event-trigger-based finite-time fuzzy adaptive control for stochastic nonlinear system with unmodeled dynamics," IEEE Transactions on Fuzzy Systems, no. 99, p. 1, 2020.

[8] Z. Wang and J. Yuan, "Fuzzy adaptive fault tolerant IGC method for STT missiles with time-varying actuator faults and multisource uncertainties," Journal of the Franklin Institute, vol. 357, no. 1, pp. 59-81, 2020.

[9] X. Chang, L. Rong, K. Chen, and W. Fu, "Lstm-based outputconstrained adaptive fault-tolerant control for fixed-wing uav with high dynamic disturbances and actuator faults," Mathematical Problems in Engineering, vol. 2021, Article ID 888, 18 pages, 2021.

[10] T. Yan, Y. Cai, and C. Wei, "Evasion-faced fast adaptive neural attitude control for generic hypersonic vehicles with structural and parametric uncertainties," Mathematical Problems in Engineering, vol. 2021, Article ID 2514073, 12 pages, 2021.

[11] A. Alatorre, E. S. Espinoza, B. Schez, P. Ordaz, and L. Garcacarrillo, "Parameter estimation and control of an unmanned aircraft-based transportation system for variablemass payloads," Asian Journal of Control, 2021.

[12] L. Kong, W. He, C. Yang, Z. Li, and C. Sun, "Adaptive fuzzy control for coordinated multiple robots with constraint using impedance learning," IEEE Transactions on Cybernetics, vol. 49, no. 8, pp. 3052-3063, 2019.

[13] V. Vajpayee, V. Becerra, N. Bausch, J. Deng, and A. J. Arul, "L1-adaptive robust control design for a pressurized watertype nuclear power plant," IEEE Transactions on Nuclear Science, vol. 68, p. 1, 2021.

[14] W. He, H. Gao, C. Zhou, C. Yang, and Z. Li, "Reinforcement learning control of a flexible two-link manipulator: an experimental investigation," IEEE Transactions on Systems, Man, and Cybernetics: Systems, no. 99, pp. 1-11, 2020. 
[15] M. Van, S. S. Ge, and H. Ren, "Robust fault-tolerant control for a class of second-order nonlinear systems using an adaptive third-order sliding mode control," IEEE Transactions on Systems, Man, and Cybernetics: Systems, vol. 47, pp. 1-8, 2016.

[16] X. Wei, Y. Zhang, Modeling and trajectory tracking control for flapping-wing micro aerial vehicles," IEEE/CAA Journal of Automatica Sinica, vol. 8, no. 1, pp. 148-156, 2021.

[17] L. Chen and Q. Wang, "Adaptive robust control for a class of uncertain mimo non-affine nonlinear systems," vol. 3, no. 1, pp. 105-112, 2016.

[18] S. Sui, C. L. P. Chen, and S. Tong, "Neural network filtering control design for nontriangular structure switched nonlinear systems in finite time," IEEE Transactions on Neural Networks and Learning Systems, vol. 30, no. 7, pp. 2153-2162, 2019.

[19] S. Sui, C. L. P. Chen, and S. Tong, "A novel adaptive NN prescribed performance control for stochastic nonlinear systems," IEEE Transactions on Neural Networks and Learning Systems, vol. 32, no. 7, pp. 3196-3205, 2021.

[20] D. Yu and C. L. P. Chen, "Automatic leader-follower persistent formation generation with minimum agent-movement in various switching topologies," IEEE Transactions on Cybernetics, vol. 50, no. 4, pp. 1569-1581, 2020.

[21] C. Zhang and Q. Dong, "Adaptive sliding mode control of fixed-wing uav considering input saturation," Aeronaut, no. S1, , 2017.

[22] H. Castaeda, O. S. Salas-Pea, and J. D. Le-Morales, "Extended observer based on adaptive second order sliding mode control for a fixed wing uav," ISA Transactions, vol. 66, p. 226, 2016.

[23] S. Kurnaz, O. Cetin, and O. Kaynak, "Adaptive neuro-fuzzy inference system based autonomous flight control of unmanned air vehicles," Expert Systems with Applications, vol. 37, no. 2, pp. 1229-1234, 2010.

[24] S. Zhao, X. Wang, D. Zhang, and L. Shen, "Model-free fuzzy adaptive control of the heading angle of fixed-wing unmanned aerial vehicles," Journal of Aerospace Engineering, vol. 30, no. 4, Article ID 04017019, 2017.

[25] J. F. Gomez and M. Jamshidi, "Fuzzy adaptive control for a uav," Journal of Intelligent and Robotic Systems, vol. 62, no. 2, pp. 271-293, 2011.

[26] Y. Xiao, Y. Fu, C. Wu, and P. Shao, "Modified model reference adaptive control of uav with wing damage," in Proceedings of the International Conference on Control, Barcelona, Spain, December 2016.

[27] W. Mackunis, Z. D. Wilcox, M. K. Kaiser, and W. E. Dixon, "Global adaptive output feedback tracking control of an unmanned aerial vehicle," IEEE Transactions on Control Systems Technology, vol. 18, no. 6, pp. 1390-1397, 2010.

[28] S. P. Bhat and D. S. Bernstein, "Finite-time stability of continuous autonomous systems," SIAM Journal on Control and Optimization, vol. 38, no. 3, pp. 751-766, 2000.

[29] J. Zhang, Q. Li, N. Cheng, and B. Liang, "Non-linear flight control for unmanned aerial vehicles using adaptive backstepping based on invariant manifolds," Proceedings of the Institution of Mechanical Engineers - Part G: Journal of Aerospace Engineering, vol. 227, 2013.

[30] S. Sui, S. Tong, and C. L. P. Chen, "Finite-time filter decentralized control for nonstrict-feedback nonlinear largescale systems," IEEE Transactions on Fuzzy Systems, vol. 26, no. 6, pp. 3289-3300, 2018.

[31] H. Castaeda, O. S. Salas-Pea, and J. D. Le-Morales, "Robust flight control for a fixed-wing unmanned aerial vehicle using adaptive super-twisting approach," Proceedings of the
Institution of Mechanical Engineers - Part G: Journal of Aerospace Engineering, vol. 228, 2014.

[32] Y. Wang, H. Sun, and L. Hou, "Event-triggered antidisturbance attitude and vibration control for t-s fuzzy flexible spacecraft model with multiple disturbances," Aerospace Science and Technology, vol. 117, Article ID 106973, 2021.

[33] Z. Su, C. Li, and Y. Liu, "Anti-disturbance dynamic surface trajectory stabilization for the towed aerial recovery drogue under unknown airflow disturbances," Mechanical Systems and Signal Processing, vol. 150, Article ID 107342, 2020.

[34] W. Yang, "On stability of linear time-varying system," IEEE Transactions on Automatic Control, vol. 21, no. 5, pp. 775-776, 2003.

[35] Q. Hu, T. Han, and M. Xin, "Analytical solution for nonlinear three-dimensional guidance with impact angle and field-ofview constraints," IEEE Transactions on Industrial Electronics, no. 99, p. 1, 2020.

[36] Q. Hu, T. Han, and M. Xin, "New impact time and angle guidance strategy via virtual target approach," Journal of Guidance, Control, and Dynamics, vol. 41, no. 8, pp. 17551765, 2018.

[37] S. P. Bhat and D. S. Bernstein, "Continuous finite-time stabilization of the translational and rotational double integrators," IEEE Transactions on Automatic Control, vol. 43, no. 5, pp. 678-682, 1998.

[38] Y. Yigwruang Hong, J. Jie Huang, and Y. Yangsheng Xu, "On an output feedback finite-time stabilization problem," IEEE Transactions on Automatic Control, vol. 46, no. 2, pp. 305309, 2001.

[39] J. Huang, W. Wang, and Y.-D. Song, "Design of adaptive finite-time controllers for nonlinear uncertain systems based on given transient specifications," Automatica, vol. 69, pp. 395-404, 2016.

[40] M. Galicki, "Finite-time control of robotic manipulators," Automatica, vol. 51, pp. 49-54, 2015.

[41] Y. Guo and S. Song, "Adaptive finite-time backstepping control for attitude tracking of spacecraft based on rotation matrix," Chinese Journal of Aeronautics, vol. 27, pp. 375-382, 2014.

[42] H. Li, S. Zhao, W. He, and R. Lu, "Adaptive finite-time tracking control of full state constrained nonlinear systems with dead-zone," vol. 100, pp. 99-107, 2019.

[43] C. Fu, Y. Tian, H. Huang, L. Zhang, and C. Peng, "Finite-time trajectory tracking control for a 12-rotor unmanned aerial vehicle with input saturation," ISA Transactions, vol. 81, pp. 52-61, 2018.

[44] Y. Yao, J. Tan, J. Wu, and X. Zhang, "Event-triggered fixedtime adaptive fuzzy control for state-constrained stochastic nonlinear systems without feasibility conditions," Nonlinear Dynamics, vol. 105, pp. 403-416, 2021.

[45] S. Zhang, S. Yuan, X. Yu, L. Kong, Q. Li, and G. Li, “Adaptive neural network fixed-time control design for bilateral teleoperation with time delay," IEEE Transactions on Cybernetics, no. 99, pp. 1-14, 2021.

[46] S. Jiang, Y. Qi, S. Cai, and X. Lu, "Light fixed-time control for cluster synchronization of complex networks," Neurocomputing, vol. 424, no. 6177, 2020.

[47] C. Wei, J. Luo, H. Dai, and G. Duan, "Learning-based adaptive attitude control of spacecraft formation with guaranteed prescribed performance," IEEE Transactions on Cybernetics, pp. 1-13, 2018.

[48] C. Wei, J. Luo, H. Dai, Z. Bian, and J. Yuan, "Learning-based adaptive prescribed performance control of postcapture space 
robot-target combination without inertia identifications," Acta Astronautica, vol. 146, pp. 228-242, 2018.

[49] J. Luo, C. Wei, H. Dai, Z. Yin, X. Wei, and J. Yuan, "Robust inertia-free attitude takeover control of postcapture combined spacecraft with guaranteed prescribed performance," ISA Transactions, vol. 74, pp. 28-44, 2018.

[50] D. Yu and C. L. P. Chen, "Smooth transition in communication for swarm control with formation change," IEEE Transactions on Industrial Informatics, vol. 16, no. 11, pp. 6962-6971, 2020.

[51] C. L. P. Chen and Z. Liu, "Broad learning system: an effective and efficient incremental learning system without the need for deep architecture," IEEE Transactions on Neural Networks \& Learning Systems, vol. 29, no. 99, pp. 10-24, 2018.

[52] J. Chen, L. Huaping, X. U. Xinying, and S. Fuchun, "Multimodal information fusion based on broad learning method," CAAI Transactions on Intelligent Systems, vol. 49, pp. 277-297.

[53] F. Shuang and C. C. L. Philip, "Fuzzy broad learning system: a novel neuro-fuzzy model for regression and classification," IEEE Transactions on Cybernetics, vol. 50, pp. 414-424, 2018.

[54] C. L. P. Chen and B. Wang, "Random-positioned license plate recognition using hybrid broad learning system and convolutional networks," IEEE Transactions on Intelligent Transportation Systems, no. 99, pp. 1-13, 2020.

[55] Z. Yunfei and C. Badong, "Least p-norm based broad learning system," pattern recognition and artificial intelligence, 2019.

[56] J. Lin, Z. Liu, C. L. Philip Chen, and Y. Zhang, "Quaternion broad learning system: A novel multi-dimensional filter for estimation and elimination tremor in teleoperation," $\mathrm{Neu}$ rocomputing, vol. 380, no. 925-2312, pp. 78-86, 2020.

[57] Y. Kong, "Hyperspectral image analysis based on width learning," Ph.D. thesis, 2019.

[58] Y. Zhongdong, T. Jingjing, and X. Yonghai, "Based on board learning system for the capacity configuration of wind and photovoltaic power generations," Electrical Measurement \& Instrumentation.

[59] S. Hochreiter and J. Schmidhuber, "Long short-term memory," Neural Computation, vol. 9, no. 8, pp. 1735-1780, 1997.

[60] X. Wang, J. Wu, C. Liu, H. Yang, and W. Niu, "Exploring lstm based recurrent neural network for failure time series prediction," Journal of Bjing University of Aeronautics and Astronautics, vol. 44, no. 4, pp. 772-784, 2018.

[61] Y. Liu, C. Yin, D. Hu, and T. Zhao, "Communication satellite fault detection based on recurrent neural network," Computer Science, vol. 47, no. 2, pp. 227-232, 2020.

[62] J. Tu, J. Gao, Z. Li, and J. Zhang, "Research on structural intelligent control algorithms based on long short-term memory networks," Journal of Huazhong University of Science and Technology, vol. 47, no. 10, pp. 1-9, 2019.

[63] Y. Tang, D. Lin, L. Cao, and Y. LIU, "Attitude control of fixed wing uav under model uncertainty and disturbance," Electronics Optics and Control, vol. 27, no. 12, pp. 85-89, 2020.

[64] X. Chang, L. Rong, K. Chen, and W. Fu, "LSTM-based outputconstrained adaptive fault-tolerant control for fixed-wing UAV with high dynamic disturbances and actuator faults," Mathematical Problems in Engineering, vol. 2021, no. 11, 18 pages, Article ID 8882312, 2021.

[65] J. Liu, B. Niu, P. Zhao, X. Li, and W. Qi, "Almost fast finitetime adaptive tracking control for a class of full-state constrained pure-feedback nonlinear systems," International Journal of Robust and Nonlinear Control, vol. 30, pp. 7517-7532, 2020.
[66] X. Ning, Y. Zhang, and Z. Wang, "Robust adaptive control for a class of T-S fuzzy nonlinear systems with discontinuous multiple uncertainties and abruptly changing actuator faults," Complexity, vol. 2020, Article ID 8863073, 16 pages, 2020 . 